\title{
Harvard und das Office of Strategic Services* Ein akademischer Beitrag zu einem freien und unabhängigen Österreich
}

\begin{abstract}
Harvard and the Office of Strategic Services
An academic contribution to a free and independent Austria

When the tide turned in favor of the Allies in the Second World War, preparations for the denazification and redemocratization of Europe began as the war progressed. These preparations posed significant challenges for the US authorities, as they had to rely on local people in the liberated areas for administrative tasks in the immediate postwar period. The Office of Strategic Services was commissioned to collect the relevant information to ensure the best possible selection of pro-democratic individuals. The OSS in turn used a group of Harvard University employees who had opposed the Nazi regime before the United States entered the war and were well networked with the OSS on the one hand and possible academic informants on the other. They worked together on the Biographical Records Project and other intelligence projects that should contribute to the liberation and re-democratization of Europe.
\end{abstract}

Keywords: Harvard University - intelligence studies - Office of Strategic Services - World War II

\section{Vorwort}

Während eines Seminars von Prof. Thomas Olechowski im Jahr 2016 über Hans Kelsen und seinen Kreis erwähnte Prof. Olechowski, dass Hans Kelsen und Josef Laurenz Kunz in den 1950er Lektoren für Völkerrecht am United States Naval War College gewesen waren. Es handelte sich um eine Ära der politischen Unterdrückung in den USA, die später als McCarthy-Ära bekannt wurde. Personen mit möglichen Verbindungen zur politischen Linken drohte vorgeworfen zu werden, Kommunist oder kommunistischer Sympathisant zu sein. Kelsen wurde immer wieder mit der politischen Linken in Verbindung gebracht, was damals für eine Ablehnung in einem sensiblen Bereich des Staatsdienstes ausgereicht hätte. Dies warf die Frage auf, ob und wie Kelsen

\footnotetext{
* Mit besonderem Dank an die Kanzlei Motamedi und Mag. Julian Motamedi für die Finanzierung meiner Forschungsreise nach Harvard, an Prof. Dr. Thomas Olechowski für seine Hilfestellung und seinen Beitrag http://dx.doi.org/10.1553/BRGOE2020-1s61
}

und Kunz das Vertrauen der US-Geheimdienste gewinnen konnten, um für eine Lehrtätigkeit in Frage zu kommen. Ebenfalls war es ein Ziel dieser Arbeit, einzelne Prozesse und Vorbereitungen der USA bezüglich einer Militärregierung im Nachkriegsösterreich, unter Beteiligung österreichischer Exilanten, zu erforschen. Eine erste Vermutung war daher, dass es möglicherweise bereits während des Zweiten Weltkriegs eine $\mathrm{Zu}$ sammenarbeit zwischen Kelsen und Kunz auf der einen und den US-Geheimdiensten auf der anderen Seite gegeben hat. Als im Jahr 2017 die Central Intelligence Agency ihre Akten online veröffentlichte, begann die Suche nach Hinweisen auf eine mögliche Verbindung zwischen Hans Kelsen, Josef Kunz und der Central Intelligence Agency sowie deren Vorgänger, dem Office of Strategic Services. Tatsächlich fanden

zu dieser Arbeit, an Verena Wagner für ihre Korrekturen sowie an die immer hilfsbereiten Mitarbeiter der Harvard University Archives. 
sich in den Geheimdienstakten die ersten Hinweise auf eine mögliche Verbindung. Obwohl die ehemals streng geheimen Akten und die darin enthaltenen Informationen anonymisiert wurden, führten darin enthaltene Hinweise zur Harvard University. Nach weiteren Recherchen verdichteten sich die Hinweise darauf, dass die Antworten auf diese Fragen in den Archiven der Harvard University gefunden werden könnten. Daher folgte im Jahr 2017 eine Forschungsreise an dieselbe, um die diesbezüglichen Akten zu sichten. Die untersuchten Akten lieferten nicht nur die erhofften Antworten, sondern dokumentieren auch den weitgehend unbekannten Widerstandskampf amerikanischer und europäischer Künstler, Intellektueller und Wissenschaftler gegen Hitlerdeutschland. Noch bevor die USA offiziell in den Krieg eintraten, engagierten sich Angestellte der Harvard University und ihre Familien und gründeten die American Defense Harvard Group. Das Ziel dieser Gruppe war der Kampf zur Befreiung Europas, die Wiederherstellung seiner Demokratie und die Verteidigung Amerikas.

\section{Einführung}

Aufgrund einer Präsidialverordnung ist der zivile Auslandsgeheimdienst der Vereinigten Staaten, die Central Intelligence Agency, verpflichtet, alle freigestellten Aufzeichnungen freizugeben. Das Archiv enthält nicht nur CIA-Akten, sondern auch die Aufzeichnungen der Vorgängerorganisation, dem Office of Strategic Services (OSS). Das OSS war der erste moderne Geheimdienst in den Vereinigten Staaten während des Zweiten Weltkriegs. Der folgende Aufsatz basiert daher hauptsächlich auf Recherchen in den online-verfügbaren Akten des CIA Crest-Programmes und auf Forschungen in den Harvard University Archives. Das OSS war sowohl ein militärischer als auch ein ziviler Geheimdienst, der in großem Ausmaß empirische Wissenschaft in seine Arbeit einbezog und mehrere hochrangige und namhafte Wissenschaftler beschäftigte. William J. Donovan, der das OSS leitete, war ein Absolvent der Harvard University und rekrutierte mehrere Mitarbeiter aus dem universitären Betrieb, um diese für seinen Geheimdienst arbeiten zu lassen. Während des Krieges arbeitete das OSS auch mit anderen Mitarbeitern der American Defense Harvard Group (ADHG) zusammen. Die ADHG führte ihre eigenen Aufzeichnungen über alle Projekte, an denen sie beteiligt war. Diese Aufzeichnungen sind nicht alle in den zugänglichen Archiven der CIA enthalten, obwohl sie eine große Anzahl von unveröffentlichten Originaldokumenten im Zusammenhang mit dem OSS umfassen. Die Aufzeichnungen der American Defense Harvard Group waren 50 Jahre lang unter Verschluss, da die Offenlegungsregeln der Harvard University dies vorschreiben, und erhielten bisher wenig Aufmerksamkeit von Wissenschaftlern. Der folgende Aufsatz behandelt nur eine begrenzte Zahl österreichischer Informanten, da die Menge an Information und beteiligten Informanten in den Unterlagen außerordentlich groß ist. Bemerkenswert ist, dass einige berühmte Persönlichkeiten, wie der Gründer der Bauhausbewegung Walter Gropius oder der Dirigent Arturo Toscanini ebenfalls als Informanten beteiligt waren. Der Fokus historischer Forschungen in Bezug auf Nachrichtendienste zielt oft auf militärische Spezialoperationen und andere Aspekte asymmetrischer Kriegsführung ab, während der nicht-operativen Sammlung und Verarbeitung von Informationen weniger Interesse geschenkt wird. Daher liefern diese Materialien immer noch eine Vielzahl unbekannter und interessanter Erkenntnisse. 


\section{American Defense Harvard Group}

\subsection{Historischer Hintergrund und Funktionsprinzipien}

Tief besorgt über die Evakuierung von Dünkirchen und den Fall Frankreichs im Juni 1940 versammelte sich eine Gruppe von Mitarbeitern der Harvard University um den Philosophieprofessor und Pulitzer-Preisträger Ralph Barton Perry, um eine ungewöhnliche akademische Kriegskoalition zu bilden. Diese trug den Namen American Defense Harvard Group. Die Mitglieder der Gruppe waren überzeugt, dass die Pläne NaziDeutschlands eine unmittelbare Bedrohung für die Vereinigten Staaten, ihre Demokratie und ihre Bürger darstellten. ${ }^{2}$ Am 24. Juni und 1. Juli 1940 fanden zwei informelle Treffen statt, die zur Gründung der ADHG führten. ${ }^{3}$ Der Hauptzweck war es, „die Verteidigungsaktivitäten jener amerikanischen Lehrer, Offiziere, Angestellten, ihrer Frauen und Familien zu fokussieren und zu lenken, die bereits durch ihre Verbindung mit der Harvard University zusammengeführt wurden; und mit ähnlichen Gruppen in anderen Gemeinschaften zusammenzuarbeiten und sie $\mathrm{zu}$ fördern. ${ }^{4}$ Die Mitglieder der ADHG waren überzeugt, dass die Achsenmächte nur mit militärischer Gewalt bekämpft werden konnten und mussten. Dies führte zu einer landesweiten Interaktion mit zahlreichen Einzelpersonen und Institutionen. Diese reichte von Privatpersonen bis hin zu Regierungsbehörden und dem Präsidenten der Vereinigten Staaten. Die ADHG war auch an den Prozessen zur Gründung des OSS beteiligt. Die Verbindung mit dem OSS und seinem

\footnotetext{
${ }^{2}$ Ralph Barton Perry, Bericht des Vorsitzenden für den Zeitraum: 24. 6. 1940-15. 9. 1940, HUA, HUD 3139 Records of the ADHG, Box 1, pag. 3.

${ }^{3}$ Ebd.

${ }^{4}$ Ebd. $3 f$.

${ }^{5}$ List of Articles by Ralph Barton Perry, HUA, HUD 3139 Records of the ADHG, Box 14.
}

Direktor William Donovan führte dazu, dass Mitglieder der ADHG direkte Mitarbeiter des OSS wurden. Diese vertiefte Verbindung führte zu mehreren OSS- und US-Regierungsprojekten, die gemeinsam mit der ADHG durchgeführt wurden. Eine dieser geheimen Tätigkeiten war das Biographical Records Project (hiernach BRP). Dieses Projekt wurde von der US-Regierung initiiert, um Informationen über mögliche zukünftige Kollaborateure für US-Streitkräfte und Beamte zu sammeln, die für die Bildung von Militärregierungen in der unmittelbaren Nachkriegszeit in Europa notwendig waren.

Als der Krieg andauerte, wuchs die anfänglich kleine Zahl der Mitglieder bis zur Auflösung der Gruppe im Juni 1945 kontinuierlich an. Zum Zeitpunkt ihrer Auflösung erreichte die ADHG mehr als 1700 Mitglieder, mit einem aktiven Aufgebot von 240 Freiwilligen. Obwohl die ADHG aus Mitgliedern der Harvard University bestand und mit staatlichen Institutionen zusammenarbeitete, blieb der Status der Gruppe informell. Die Gruppe veröffentlichte Artikel ${ }^{5}$ in amerikanischen Zeitungen und forderte die Regierung der Vereinigten Staaten und ihren Präsidenten auf, das Vereinigte Königreich aktiver zu unterstützen und in den Krieg gegen Deutschland einzutreten. Durch diese versuchte Einflussnahme zog die ADHG die Aufmerksamkeit offizieller Regierungsinstitutionen auf sich. ${ }^{6}$ Die Analyse der militärischen und politischen Lage in Europa durch die ADHG fiel mit den Erkenntnissen des späteren OSS-Direktors William Donovan zusammen. Dieser berichtete während seines Studiums des britischen Geheimdienstes (SIS) Präsident Roosevelt, dass Großbritannien dringend Hilfe benötige. ${ }^{7}$ Im Jahr 1941 unterstützte Ralph Barton Perry den designierten OSS-Direktor William Donovan

\footnotetext{
${ }^{6}$ Ralph Barton Perry an Präsident Franklin Delano Roosevelt, 28. 6. 1941, HUA, HUD 3139 Records of the ADHG, Box 11.

${ }^{7}$ MCINTOSH, Sisterhood of Spies 5.
} 
bei der Auswahl von Akademikern, die für die Bildung seines neuen Geheimdienstes benötigt wurden. ${ }^{8}$ Mitglieder der ADHG und andere Akademiker schlossen sich so dem ersten modernen USGeheimdienst an, indem sie unmittelbare Mitarbeiter im intellektuellen Rückgrat des OSS - der Research and Analysis Branch - wurden. ${ }^{9}$ Diese Akademiker arbeiteten projektbezogen während des Krieges mit der ADHG weiterhin zusammen. Neben der Zusammenarbeit mit dem OSS arbeitete die ADHG auch mit mehreren anderen USRegierungs- und Militärinstitutionen zusammen und leistete einen wissenschaftlichen Beitrag zu einer Reihe militärischer und nachrichtendienstlicher Kriegsprojekte. Diese reichten von der militärischen Ausbildung über wissenschaftliche Analysen und Berichte bis hin zum Schutz europäischer Denkmäler.

Wie der OSS-Direktor William Donovan war auch die ADHG überzeugt, dass nur eine starke Unterstützung Großbritanniens den Kriegsverlauf positiv beeinflussen könne und als Folge davon die erste wichtige Konfrontation die Atlantikschlacht sei. ${ }^{10}$ Daher mussten alle notwendigen Maßnahmen ergriffen werden, um weiterhin eine angemessene Versorgung Großbritanniens mit Waffen und Vorräten zu gewährleisten. Großbritannien wurde von der ADHG als „das Hauptbollwerk zwischen der Achse und der westlichen Hemisphäre" betrachtet. ${ }^{11}$ Vor dem Angriff auf Pearl Harbour war die Stimmung in der amerikanischen Öffentlichkeit von einer Abneigung gegen einen Kriegseintritt dominiert. Dieser Umstand war der ADHG wohlbekannt, weshalb auch Zeitungsartikel veröffentlicht wurden, um dieser Meinung gegenzusteuern. So setzte sich die ADHG bereits 1940 mit Fragen der

\footnotetext{
${ }^{8}$ Ralph Barton Perry an William Joseph Donovan, 14. 7. 1941, HUA, HUD 3139 Records of the ADHG, Box 5 .

${ }^{9}$ TRAUSSNIG, Militärischer Widerstand 210.

${ }^{10}$ Ralph Barton Perry an Franklin Delano Roosevelt, 28. 6. 1941, HUA, HUD 3139 Records of the ADHG, Box 11.
}

Bewusstseinsbildung und dem öffentlichen Diskurs auseinander und ergriff Maßnahmen zur Beeinflussung in diesen Bereichen.

\subsection{Struktur der American Defense Harvard Group}

Unmittelbar nach ihrer Gründung 1940 nahm die ADHG ihre Tätigkeit auf und richtete verschiedene Komitees mit besonderem Sachbezug ein. Das Hauptelement der ADHG war das Lenkungskomitee, das sich aus den Generaloffizieren der Gruppe und den Vorsitzenden der Sonderausschüsse zusammensetzte. Dieses Steuerungselement hielt wöchentliche Sitzungen ab, um das weitere Vorgehen zu planen und zu koordinieren. ${ }^{12}$ Bereits im Lenkungsausschuss zeigte sich ein Österreichbezug, denn eines seiner Mitglieder war der österreichische Kunsthistoriker Otto Benesch. Dieser spielte auch eine wichtige Rolle im BRP und beim Beitrag der Gruppe für den Denkmalschutz in Europa. Die Generalversammlung der Gruppe bestand aus einem Vorsitzenden, elf Beratern, vier stellvertretenden Vorsitzenden, einem Sekretär und einem Schatzmeister. Jedes gegründete Komitee wurde einer von vier Abteilungen der ADHG untergeordnet. Diese vier Sektionen und ihre Ausschüsse waren wie folgt aufgebaut:

1. (Leidens-)Linderung, unter Einbeziehung des Ausschusses für die Betreuung europäischer Kinder und des Ausschusses für medizinische Dienste;

2. Öffentlichkeitsarbeit, die die Ausschüsse für Information, Presse und Schreiben, Radio und Sprechen, Politik, Friedensregelung, Arbeit und Industrie, Produktion und panamerikanische Beziehungen umfasste;

\footnotetext{
${ }^{11}$ Ebd.

${ }^{12}$ Ralph Barton Perry, Bericht des Vorsitzenden für den Zeitraum: 24. 6. 1940-15. 9. 1940, HUA Archive, HUD 3139 Records of the ADHG, Box 1, pag. 3.
} 
3. Ausbildung, Einbeziehung der Ausschüsse für militärische Ausbildungslager, Marineausbildung, Ausbildung an der Universität und Ausbildung für den staatlichen Dienst;

4. Moral und Korrespondenz, die die Ausschüsse für nationale Moral, Jugendprobleme und Korrespondenz umfasste. ${ }^{13}$

\subsection{ADHG-Ausschüsse und -Projekte}

Aufgrund der großen Anzahl von Projekten und Aktivitäten, an denen die ADHG beteiligt war oder die von ihr durchgeführt wurden, kann in diesem Aufsatz nur ein kleiner Bruchteil genauer beschrieben werden. Die große Vielfalt an Projekten sollte dazu beitragen, den Krieg zu gewinnen und helfen, mögliche Probleme der Nachkriegszeit zu lösen. Die ADHG wollte nicht nur die USA und ihre Bürger schützen, sondern auch Europa vom Faschismus befreien und seine Demokratie wiederherstellen. Diese Bemühungen erforderten unorthodoxe Ansätze, die sowohl Mitarbeiter als auch Studenten betrafen und zu einer teilweise den Kriegserfordernissen angepassten Universitätslehre führten. Mit der voranschreitenden Dauer des Krieges wurde das Leben an der Harvard University mehr und mehr vom Krieg und seinen Anforderungen durchdrungen. So spielten ab dem Jahr 1941 die kriegsbedingten Aktivitäten eine immer größer werdende Rolle im täglichen Leben der Universität.

\section{Denkmalschutz}

1943 schlug die ADHG Pläne für die Einrichtung einer „offiziellen Kommission zur Vermessung

\footnotetext{
${ }^{13}$ Ebd.

${ }^{14}$ Ralph Barton Perry an Donald McKay, 24. 12. 1942, HUA, HUD 3139 Records of the ADHG, Box 9.

${ }^{15}$ Vertrauliche Veröffentlichung, Hauptquartier der Alliierten Streitkräfte G-5 Abschnitt APO 512, 28. 5. 1944, HUA, HUD 3139 Records of the ADHG, Box 73. ${ }^{16}$ Ebd.

${ }^{17}$ Otto Benesch, Long List of Monuments for Austria, HUA, HUD 3139 Records of the ADHG, Box 73; Otto und Eva Benesch, Long List of Monuments Czechoslovakia, HUA, HUD 3139 Records of ADHG, Box 73.
}

und zum Schutz von Kunstwerken, Denkmälern etc." im umkämpften Europa vor. ${ }^{14}$ Die ADHG wurde zu diesem Thema von Professor Sachs und Herrn Stout W. G. Constable vom Boston Museum of Fine Arts kontaktiert. ${ }^{15}$ Ziel des Denkmalschutzes war nicht nur die Rettung des kulturellen Erbes selbst, sondern auch die Verhinderung schädlicher Auswirkungen auf die zivile Moral, die durch den Verlust dieser Denkmäler befürchtet wurde. ${ }^{16}$ Mehrere Kunsthistoriker im Exil trugen zu diesem Projekt bei, indem sie Listen von Denkmälern in Europa erstellten. Die Harvard University und ihr Umfeld verfügten durch die hohe Anzahl an Exilanten über die wissenschaftlichen Humanressourcen, die für die Ausarbeitung dieser Listen erforderlich waren. Der österreichische Kunsthistoriker Otto Benesch und seine Frau Eva trugen mit der Erstellung von Denkmallisten für Österreich und die Tschechoslowakei zu dem Projekt bei. ${ }^{17}$ Die Arbeit des Ehepaares war laut Aktenlage von höchster Qualität und wurde von den Behörden in Washington äußerst positiv bewertet. ${ }^{18}$ Die erstellten Listen wurden unter anderem für Handbücher der militärischen Besatzungstruppen verwendet. ${ }^{19}$

\section{Industrial Management Engineering}

Um die Volkswirtschaft an die Bedürfnisse einer Kriegsindustrie anzupassen, wurde ein spezieller Universitätslehrgang der Harvard Business School angeboten. Dieser bestand aus einem zwölfmonatigen Programm an der Graduate School of Business Administration. ${ }^{20}$ Ziel dieses Lehrganges war es, Personen „für Unternehmen

\footnotetext{
${ }^{18}$ ADHG an Otto und Eva Benesch, 7. 9. 1943, HUA, HUD 3139 Records of ADHG, Box 72.

${ }^{19}$ Ebd. 4.

${ }^{20}$ Industrial Management Engineering, Ein zwölfmonatiger Studiengang zum Abschluss des Industrieadministrators, HUA, HUD 3139 Records of the ADHG, Box 31, pag. 1.
} 
auszubilden, die wichtige Munition und Vorräte herstellten", aber auch für die amerikanischen Streitkräfte direkt. ${ }^{21}$ Dieses Sonderprogramm versuchte die Probleme zu lösen, die sich aus "dem Mangel an ausgebildeten Arbeitskräften ergaben, die für die Erfüllung der Anforderungen einer stärkeren Steigerung der industriellen Produktion" nötig waren, und sollte dazu beitragen, „den Bedürfnissen der Durchführung eines aggressiven Krieges gerecht zu werden“. ${ }^{22}$

\section{Komitee für Militärische Trainingslager und Harvard Military Camp}

Akademiker aus Harvard unterrichteten während des Krieges auch Soldaten und OSS-Agenten. Dazu gehörte unter anderem auch eine Ausbildung in psychologischer Kriegsführung. Zukünftige OSS-Agenten wurden psychologischen Tests, simulierten Verhören und anderen Übungen unterzogen. ${ }^{23}$ Diese sollten vorsätzlich zu einer psychologischen Eskalation führen, um die geistige Belastbarkeit der potentiellen Agenten $\mathrm{zu}$ testen. ${ }^{24}$ Österreichische Auszubildende, die den europäischen Faschismus bereits persönlich erlebt hatten, hielten einige dieser Methoden anfänglich für unrealistisch oder sogar gefährlich, wenn diese praktisch angewendet würden. Es gab die Befürchtung durch die angehenden Agenten, dass diese Methoden „höchstwahrscheinlich dazu führen würden, dass sie gefangen genommen und erschossen werden “. ${ }^{25}$

Es wurde zunehmend klar, dass die industriellen Anstrengungen durch die USA allein nicht ausreichen würden, um Europa und die Welt zu retten. Deshalb mussten weitere Vorbereitungen für

\footnotetext{
${ }^{21}$ Ebd.

${ }^{22}$ Ebd.

${ }^{23}$ Ralph Barton Perry, Bericht des Vorsitzenden für den Zeitraum: 24. 6. 1940-15. 9. 1940, HUA, HUD 3139 Records of the ADHG, Box 1, pag. 15.

${ }^{24}$ TRAUSSNIG, Militärischer Widerstand 230.

${ }^{25}$ Ebd.

${ }^{26}$ Alfred Chester Hanford, Appeal for Proposed Harvard Military Camp, HUA, HUD 3139 Records of the ADHG, Box 31.
}

junge Amerikaner getroffen werden, darunter auch Studenten und Absolventen, damit diese aktiv in den Kriegsdienst eintreten konnten. $\mathrm{Ab}$ 1941 lud der Dekan des Harvard College daher Studenten und Absolventen zu einem militärischen Sommercamp in das Squam Lake Camp in New Hampshire ein. ${ }^{26}$ Während des sechswöchigen Kurses wurden die Teilnehmer in Kartenlesen, Topographie und Vermessung geschult. ${ }^{27}$ Seit 1942 druckte und veröffentlichte die Harvard University ein offizielles Informationsblatt zum Thema „Möglichkeiten für spezielle Ausbildungen während des Krieges". In diesem wurde darauf hingewiesen, dass Männer, die in Physik und Ingenieurwissenschaften ausgebildet wurden, am meisten von Nöten waren. Andere Kurse umfassten auch Astronomie, Biologie, Ökonomie, bildende Kunst, Geologie, Geschichte, Sprachen, Mathematik, Physik und Psychologie. ${ }^{28}$

\section{Ausschuss für die Betreuung europäischer Kinder}

Dieser Ausschuss konzentrierte sich auf die Unterstützung von Flüchtlingskindern aus Großbritannien und anderen europäischen Ländern. ${ }^{29}$ Das Komitee diente als Zweigstelle des United States Committee for Care of European Children mit Sitz in New York. Es wurde Geld gesammelt, um die Kosten und Garantien für den Unterhalt der Kinder, für die Pflegefamilien zur Verfügung gestellt wurden, zu decken.

\section{Ausschuss für Moral und nationale Einheit}

Der Zweck dieses Komitees war es, „ein Gefühl der Hingabe an Amerika und die amerikanischen

\footnotetext{
${ }^{27}$ Ebd.

${ }^{28}$ Offizielles Register der Harvard University Bd. XXXIX, Opportunities for Special Training during the War, 20. 1. 1942, HUA, HUD 3139 Records of the ADHG, Box 31, pag. 3f.

${ }^{29}$ Ralph Barton Perry, Bericht des Vorsitzenden für den Zeitraum: 24. 6. 1940-15. 9. 1940, HUA, HUD 3139 Records of the ADHG, Box 1, pag. 5.
} 
Institutionen zu fördern, Meinungsverschiedenheiten zu heilen, die Apathischen und Unentschlossenen wachzurütteln und die militärische Bereitschaft unter Berücksichtigung der menschlichen und sozialen Werte, die damit verbunden sind, in Einklang zu bringen". ${ }^{30}$ Das Komitee erstellte eine Erklärung zur amerikanischen Moral, die an alle Mitglieder des Kongresses und an andere Personen in wichtigen Regierungspositionen versandt wurde. ${ }^{31}$ Die Mitglieder der ADHG betrachteten einen Krieg mit den Achsenmächten als eine unvermeidliche Notwendigkeit - eine Meinung, die in der Öffentlichkeit in den USA anfänglich nicht geteilt wurde. In diesem Zusammenhang schloss sich die ADHG mit Edward Bernays zusammen, einem der führenden Propagandaexperten des 20. Jahrhunderts und Neffen von Sigmund Freud, der dessen psychoanalytische Forschung für seine Arbeit verwendete. Edward Bernays und Ralph Barton Perry nahmen auch gemeinsam an einem Treffen zu Propagandafragen in New York teil. ${ }^{32}$

\section{Kriegsgefangene}

Die ADHG versuchte auch (österreichischen) Kriegsgefangenen in den USA zu helfen, die unter einer „Lager-Gestapo" in ihren Gefängnissen litten. ${ }^{33}$ Die Austrian Action, das Free Austrian Movement und andere österreichische Exilorganisationen forderten die US-Regierung immer wieder auf, deutsche und österreichische Gefangene zu trennen. Aber eine anhaltende bürokratische Kontroverse über die Zuständigkeit in dieser Angelegenheit verhinderte eine Verbesserung der Situation. ${ }^{34}$ Ein Memorandum über die Entlastung der Kriegsgefangenen veranschaulicht die Probleme, mit denen österreichische

\footnotetext{
${ }^{30}$ Ebd. 15.

${ }^{31}$ Ebd.

${ }^{32}$ Edward Bernays an Ralph Barton Perry, 11. 9. 1940, HUA, HUD 3139 Records of the ADHG, Box 4.

${ }^{33}$ EPPEL, Österreicher im Exil 30.

${ }^{34}$ Ebd. 31.

${ }^{35}$ STIFTER, Zwischen geistiger Erneuerung und Restauration 230 .
}

Kriegsgefangene konfrontiert waren, wenn sie unter dem Terror anderer Kriegsgefangener standen, die noch an den vermeintlichen Endsieg glaubten. ${ }^{35}$

\section{Ausschuss für Probleme der Friedensregelung}

Die Ergebnisse des internationalen Friedensregelungsprozesses nach dem Ersten Weltkrieg waren offensichtlich alles andere als erfreulich. Während sich die meisten Ausschüsse hauptsächlich auf Probleme von unmittelbarer Bedeutung für den Kriegsverlauf konzentrierten, fokussierte sich dieser Ausschuss in erster Linie auf die Aufrechterhaltung von Frieden und Demokratie im Europa der Nachkriegszeit. Sidney Fay war der Vorsitzende des Komitees und erklärte, dass „im Ersten Weltkrieg die Alliierten den Krieg zwar gewonnen haben, aber den Frieden verloren haben."36 Dieser Ausschuss zielte wie viele andere Gruppierungen in den Vereinigten Staaten darauf $a b$, den dauerhaften Wandel zu stabilen und demokratischen Verhältnissen in der Nachkriegszeit zu sichern. ${ }^{37}$ Der aus Österreich stammende Akademiker Gottfried Haberler war Mitglied des Komitees und partizipierte auch beim BRP (siehe unten).

\section{4 Österreichische Akademiker und ihre Verbindung zur ADHG}

Wie bereits erwähnt, gehörten mehrere österreichische Akademiker zu den Mitgliedern der ADHG. Dies ist nicht weiter verwunderlich, wenn man bedenkt, dass in den 1930/40er Jahren nicht wenige Mitglieder der österreichischen Gelehrtenwelt in die Vereinigten Staaten emigrierten und sich unter anderem an der Harvard Uni-

\footnotetext{
${ }^{36}$ Bericht des Vorsitzenden für den Zeitraum: 15.9. 1940-15. 3. 1941, HUA, HUD 3139 Records of the ADHG, Box 1, pag. 20.

${ }^{37}$ Ebd.
} 
versity niederließen. Der durch Ralph B. Perry regelmäßig veröffentlichte Report of the Chairman gibt einen Überblick über die Aktivitäten der ADHG. Auf den letzten Seiten der Berichte wurde ein Dienst- und Organisationsplan der ADHG angeführt. ${ }^{38}$ Dieser Plan stellte die organisatorische Ausformung der Organisation und deren personellen Strukturen dar.

In diesem Plan wurde unter der Rubrik der Executive Office Volunteers der österreichische Wirtschaftswissenschaftler Gottfried Haberler geführt. Er war, wie bereits erwähnt, auch ein Mitglied des Komitees für Probleme der Friedensregelung.

Eine weitere österreichische Akademikerin, die für die ADHG arbeiten wollte, war die aus Wien stammende Hedwig Schleiffer. In einem Brief vom November 1941 an Ralph B. Perry umschrieb sie ihre gegenwärtige Arbeit an der Harvard College Library. Sie schilderte in ihrem Schreiben, dass sie an der Harvard University nicht in einer Position eingesetzt werden konnte, die ihren Qualifikationen entsprach. ${ }^{39}$ Die Antwort von Ralph Barton Perry spiegelt das knappe, spendenabhängige Budget der ADHG wieder, weshalb der Antrag aufgrund unzureichender finanzieller Mittel abgelehnt wurde. ${ }^{40}$ Ein sehr auffälliger und mysteriöser deutsch-österreichischer Akademiker, der mit der Gruppe in Kontakt stand, war Karl Borromäus Frank alias Paul Hagen. ${ }^{41}$ Er wurde von Ralph B. Perry schriftlich kontaktiert, zu einem Abendessen im Harvard Faculty Club eingeladen und von die-

\footnotetext{
${ }^{38}$ Bericht des Vorsitzenden für den Zeitraum: 15.9. 1940-15. 3. 1941, HUA, HUD 3139 Records of the ADHG, Box 1, pag. 34 .

${ }^{39}$ Hedwig Schleiffer an Ralph Barton Berry, 26.11. 1941, HUA, HUD 3139 Records of the ADHG, Box 11.

${ }^{40}$ Ralph Baron Berry an Hedwig Schleiffer, 20.11. 1941, HUA, HUD 3139 Records of the ADHG, Box 11.

${ }^{41}$ Ralph Barton Perry an Paul Hagen, 8. 2. 1944, HUA, HUD 3139 Records of the ADHG, Box 7; Hagen nach Perry, 28. 2. 1944, HUA, HUD 3139 Records of the ADHG, Box 7.
}

sem speziell gebeten, am BRP mitzuwirken. ${ }^{42} \mathrm{Ha}-$ gen arbeitete auch direkt für den Coordinator of Information (hiernach COI) sowie das OSS und wurde 1942 mit dem OSS-Agenten Allen Dulles in New York gesehen. Hagen, der bereits über wichtige Erfahrungen in subversiven und konspirativen Angelegenheiten verfügte, skizzierte ein Konzept für eine Labour Section innerhalb des COI/OSS. Dies wurde 1942 als eine Unterabteilung der OSS Secret-Intelligence-Branch (OSS/SI) realisiert. ${ }^{43}$ Das anhand der Unterlagen augenscheinlich aktivste österreichische Mitglied der ADHG und gleichzeitig auch der produktivste österreichische Berater sowie Quelle des BRP war Otto Benesch. Der Vertreter der Wiener Schule der Kunstgeschichte verbrachte die Kriegsjahre in den USA und arbeitete unter anderem im Harvard Fogg Museum, welches direkt gegenüber dem Gebäude gelegen ist, das 1941 als Hans Kelsens Adresse in Harvard verzeichnet wurde. ${ }^{44}$ Otto Benesch empfahl auch Ferdinand Czernin (siehe unten) - den Vorsitzenden der Austrian Action - als wichtigen Ansprechpartner für Ralph Barton Perry. ${ }^{45}$

\section{Die American Defense Harvard Group und ihr Bezug zu österreichischen Angelegenheiten}

Abgesehen von den oben genannten österreichischen Akademikern, die ADHG-Mitglieder oder anderweitig mit der Gruppe verbunden waren, lebten in den 1940er Jahren insgesamt 1,2 Millionen Menschen mit einer engen (Ahnen-) Beziehung zu Österreich - aufgrund von mindestens einem Elternteil, der in Österreich geboren

\footnotetext{
${ }^{42}$ Ralph Barton Perry an Paul Hagen, 2. 2. 1944, HUA, HUD 3139 Records of the ADHG, Box 7.

${ }^{43}$ TRAUSSNIG, Militärischer Widerstand 232f.

${ }^{44}$ Ralph B. Perry an Hans Kelsen, 1. 12. 1941, HUA, HUD 3139 Records of the ADHG, Box 8.

${ }^{45}$ Otto Benesch an Ralph Barton Perry, HUA, HUD 3139 Records of the ADHG, Box 5.
} 
wurde oder sie selbst in Österreich geboren wurden - in den USA. ${ }^{46}$ Die ADHG pflegte daher auch mit externen österreichischen Personen Kontakt und arbeitete mit mehreren österreichischen politischen Exilgruppen zusammen. ${ }^{47}$ Infolgedessen findet sich in den Akten der ADHG eine ganze Reihe von Dokumenten mit direktem Bezug zu österreichischen Agenden. Die Aufzeichnungen der ADHG enthalten beispielsweise eine Briefsammlung ${ }^{48}$ zwischen dieser und der Austrian Action, einen Bericht über die Ereignisse rund um Otto Habsburgs 30. Geburtstag in New York, einen Ordner der Österreichischen Aktion zur Moskauer Deklaration sowie die Briefe der österreichischen Informanten und Berater an das Komitee des BRP und andere Dokumente. Diese Dokumente zeigen auch die schwierige Situation, in der sich die verschiedenen oppositionellen österreichischen Gruppen untereinander befanden. Ein Bericht von Ferdinand Czernin anlässlich der Feierlichkeiten zu Otto Habsburgs 30. Geburtstag zeigt die stark monarchistische Ausrichtung des Jubilars und wie offen sie von ihm und seinen Anhängern gezeigt wurde. ${ }^{49}$ Auf der anderen Seite engagierten sich die Austrian Action und ihr Vorsitzender Ferdinand Czernin vehement gegen undemokratische und monarchisch-restaurative Pläne für ein Nachkriegsösterreich. ${ }^{50}$

\footnotetext{
${ }^{46}$ STIFTER, Zwischen Geistiger Erneuerung und Restauration 157.

${ }^{47}$ S.W. Gould, Monarchie oder Republik in Österreich? HUA, HUD 3139 Records of the ADHG, Box 18; Schreiben der österreichischen Aktion; Bericht über die Dinner Party zu Ehren von Otto Habsburgs dreißigster Geburtstagsparty, 20. 11. 1942, HUA, HUD 3139 Records of the ADHG, Box 18.

${ }^{48}$ Ferdinand Czernin zu Ralph Barton Perry, 11. 12. 1943, HUA, HUD 3139 Records of the ADHG, Box 5; Ferdinand Czernin an Sara Wambaugh, 26. 1. 1944, HUA, HUD 3139 Records of the ADHG, Box 48.

${ }^{49}$ Dem Bericht zufolge schmückte die schwarz-gelbe Flagge der österreichischen Monarchie auch die Vorderseite des Hotelsaals und die österreichische Kaiserhymne wurde während der Veranstaltung mindestens zweimal gesungen.
}

\section{Das Office of Strategic Services und das Biographical Records Project}

\subsection{Das OSS: Historischer Hintergrund}

Das OSS wurde vom US-Oberbefehlshaber Franklin D. Roosevelt am 13. Juni 1942 gegründet. ${ }^{51}$ Es wird als Vorgänger der Central Intelligence Agency und militärischer Spezialeinheiten in den USA gewürdigt. Das Hauptziel des OSS war jedoch die Sammlung und Analyse strategischer kriegsrelevanter Informationen. ${ }^{52}$ Sein Leiter war William J. Donovan, ein konservativer New Yorker Anwalt und hochdekorierter Veteran des Ersten Weltkriegs. Er wurde von Präsident Franklin D. Roosevelt zum Leiter des OSS ernannt. ${ }^{53}$ Vor dessen offizieller Gründung war Donovan bereits als Leiter des Vorläuferdienstes - COI - tätig gewesen. Wie bereits erwähnt, arbeitete Donovan auch an der Errichtung des OSS und stand in personellen Fragen diesbezüglich in Kontakt mit der ADHG. Er konsultierte Ralph Barton Perry und Charles H. Taylor zu möglichen Beratern mit akademischem Hintergrund, um diese für das COI und OSS zu rekrutieren. ${ }^{54}$ Die ausgewählten Akademiker stellten daher während des Krieges eine direkte Verbindung zwischen dem OSS und der ADHG her. ${ }^{55}$ Das OSS war in mehrere verschiedene Abteilungen unterteilt, mit einer Vielzahl

\footnotetext{
${ }^{50}$ TRAUSSNIG, Militärischer Widerstand 43.

${ }^{51}$ Kopie des Executive/Military Order von Franklin D. Roosevelt, Oberbefehlshaber, der die Einrichtung des Office of Strategic Services anordnete (https://www.cia.gov/library/readingroom/docs/CIARDP83-01034R000200090002-9.pdf) (13. 06. 1942/14. 8. 2018)

${ }^{52} \mathrm{Ebd}$.

${ }^{53}$ TRAUSSNIG, Militärischer Widerstand 208.

${ }^{54}$ Sammlung von Korrespondenz zwischen OSS und ADHG, HUA, HUD 3139 Records of the ADHG, Box 9. ${ }^{55}$ Charles H. Taylor an William Joseph Donovan, 17. 7. 1941, HUA, HUD 3139 Records of the ADHG, Box 5; William Donovan an Charles H. Taylor, 19. 7. 1941, HUA, HUD 3139 Records of the ADHG, Box 5.
} 
von Einzelaufgaben. Diese umfassten das Sammeln und Verarbeiten aller Arten von Informationen, aber auch militärische Spezialoperationen hinter feindlichen Linien. Das BRP wurde von der Research and Analysis Branch durchgeführt. Diese Abteilung spielte innerhalb des Geheimdienstes eine wichtige Rolle. ${ }^{56}$

\subsection{Das CIA CREST-Programm und die Sammlung der American Defense Har- vard Group}

Die Central Intelligence Agency sowie andere Behörden in den Vereinigten Staaten sind gesetzlich verpflichtet, alle nicht mehr relevanten Aufzeichnungen, die 25 Jahre alt oder älter sind, automatisch zu veröffentlichen. Im Januar 2017 wurde die Sammlung des CIA Crest-Programms zusätzlich online veröffentlicht und über das Internet zugänglich gemacht. Vor 2017 mussten Forscher persönlich zu den National Archives anreisen. Teile dieser Akten sind in einzelne themenbezogene Sammlungen unterteilt, zu denen auch eine eigene OSS-Sammlung gehört. Die OSS-Sammlung umfasst auch die biografischen Dateikarten mit den Angaben österreichischer Informanten wie Hans Kelsen. ${ }^{57}$ Die Dateikarten geben zwar nicht die Namen ihrer Autoren preis, enthalten aber das Datum und den Ursprungsort bei der Erstellung. So wurde beispielweise die biografische Aufzeichnung H-1661 im Oktober 1943 in Berkeley von einem Informanten $49^{58}$ erstellt und beinhaltet Informationen über Adolf Merkl. Jede dieser Quellen war mit einem Berater verbunden, der auch auf der Dateikarte verschlüsselt notiert wurde. Diese Berater waren oft selbst einfache Informanten, wurden aber durch Nennung möglicher weiterer Informanten in den USA zusätzlich

\footnotetext{
${ }^{56}$ TRAUSSNIG, Militärischer Widerstand 210.

${ }^{57}$ OSS-Sammlung, CIA.

${ }^{58}$ Hans Kelsen, OSS Biographical Record H-1661 on Adolf Merkl 4,

www.cia.gov/library/readingroom/docs/CIA-RDP8200038R1000190017-4.pdf

(19. 10. 1943/14. 8. 2018).
}

als Berater geführt. Diese Angaben über die Berater waren mit dem Zusatz "American Defense Harvard Group" versehen. Die an die ADHG übertragene Sammlung der Daten über Akademiker und Künstler umfasste auch die Aufgabe der erstmaligen Kontaktaufnahme zu möglichen Informanten und Beratern in den USA. Dies geschah durch Korrespondenz oder durch persönliche Besuche und dabei durchgeführte Interviews. Die Briefe, Namenslisten und anderen Dokumente im Zusammenhang mit dem BRP wurden von der ADHG archiviert. Diese Materialien sind insofern einzigartig, da sie nicht in den OSSDateien des CIA-Crest-Programms auftauchen und überhaupt nicht geschwärzt wurden. Insbesondere die Sammlung der Korrespondenz zwischen der ADHG und den möglichen Informanten bietet interessante Einblicke auf einer persönlichen Ebene. Die ADHG-Akten stehen der Forschung erst seit 1990 zur Verfügung, da an der Harvard University eine Sperrfrist bis zur Veröffentlichung von fast 50 Jahren gilt. Es scheint daher, dass die Akten zum Zeitpunkt ihrer Veröffentlichung bereits in Vergessenheit geraten waren.

\subsection{Zivile Angelegenheiten und Militärregierung}

Die Bemühungen, verbündete Institutionen und Einzelpersonen auf die Nachkriegsaufgaben vorzubereiten, wurden von der Civil Affairs Division des US-Kriegsministeriums betrieben. ${ }^{59}$ Ein wichtiges Ziel der militärischen Besatzung war es, zur Wiederherstellung eines freien und unabhängigen Österreichs beizutragen. ${ }^{60} \mathrm{Um}$ dieses Ziel zu erreichen wurde unter anderem auch das

\footnotetext{
${ }^{59}$ Wilmarth S. Lewis an Ralph B. Perry, 4. 6. 1943, HUA, HUD 3139 Records of the ADHG, Box 44.

${ }^{60}$ Österreich. Military Government, Handbook for Military Government, Annex No. 32B.
} 
BRP initiiert. Sowohl das amerikanische Außenministerium als auch das Kriegsministerium befassten sich mit Fragen der Zivil- und Militärregierung in Österreich. Die damit verbundenen Themenbereiche reichten von der Entnazifizierung über den Wiederaufbau des Bildungswesens bis hin zu allen erdenklichen Fragen im Zusammenhang mit einer möglichen Militärregierung. ${ }^{61}$ Die archivierten Dokumente über die Arbeiten zu diesen Themenbereichen und ihre Ergebnisse sind unübersichtlich und zersplittert. Dies ist dem damaligen Gewirr an Kompetenzen, Abteilungen verschiedener Ministerien und der Vielzahl parallellaufender, aber nicht gemeinsam koordinierter Projekte geschuldet. ${ }^{62}$

In Angelegenheiten der Militärregierung war Kelsen nicht nur am BRP beteiligt, sondern wirkte zu österreichischen Fragen auch beim Civil Affairs Guides Program mit. ${ }^{63}$

In einem Dankschreiben an William Donovan drückte der US-Kriegsminister Henry Stimson seine persönliche Wertschätzung für den großartigen Beitrag durch das OSS aus und lobte die ausgezeichnete Zusammenarbeit im Bereich der Civil Affairs. ${ }^{64}$ Stimson weist in seinem Schreiben auch darauf hin, dass diese Zusammenarbeit einen wichtigen Faktor für eine erfolgreiche Militärregierung darstellte.

\subsection{Biographical Records Project}

Der Startschuss für das Sammeln und Verarbeiten des mit der ADHG verbundenen Teils des

\footnotetext{
${ }^{61}$ STIFTER, Zwischen Geistiger Erneuerung und Restauration 398f.

${ }^{62}$ Ebd. 220f.

${ }^{63}$ EPPEL, Österreicher im Exil 2214.

${ }^{64}$ Henry Stimson an William Donovan, https://www.cia.gov/library/readingroom/docs/CIARDP13X00001R000100370003-1.pdf,

(23. 11. 2013/14. 8. 2018).

${ }^{65}$ Wilmarth S. Lewis an Ralph Barton Perry, 4. 6. 1943, HUA, HUD 3139 Records of ADHG, Box 44.

${ }^{66}$ Wilmarth S. Lewis an Ralph Barton Perry, 4. 6. 1943, HUA, HUD 3139 Records of the ADHG, Box 44.

${ }^{67}$ McInTOSH, Sisterhood of Spies 79.
}

BRP, fiel am 8. Juni 1943. ${ }^{65}$ Auf Ersuchen des OSS und des U.S. War Departments wurde das BRP, ergänzend zu anderen Bereichen der Nachkriegsplanung, ins Leben gerufen. Es war auf mehrere Niederlassungen verteilt, die sich in zentralen Städten (Cambridge M.A., New York und Washington, D.C.) an der Ostküste der Vereinigten Staaten befanden und auf jeweils unterschiedliche gesellschaftliche Schichten abzielten. ${ }^{66}$ Die New York Biographical Records Section nahm überhaupt als eine der ersten Secret Intelligence (SI) Branches des OSS ihre Arbeit auf und wurde ab 1941 von Emmy Crisler Rado geleitet. ${ }^{67}$ Diese Sektion fokussierte sich auf Informationen von normalen Immigranten und sammelte hauptsächlich allgemeine Informationen. Bis 1941 hieß diese OSS-Niederlassung Oral Intelligence Branch. ${ }^{68}$ Zwischen Juni 1943 und Juni 1944 befragte diese Sektion 706 Österreicher. ${ }^{69}$

Die von der ADHG gesammelten Informationen über Akademiker und Künstler wurden an das OSS-Büro in Washington weitergeleitet. ${ }^{70}$ Das primäre Ziel der Erhebung wird in den Dokumenten in Harvard wie folgt angegeben: ,[...] so genaue und aktuelle Informationen wie möglich über Personen zu erhalten, die in der Zeit unmittelbar nach den Feindseligkeiten von Nutzen sein könnten. " 71 Dem BRP wurde ,eine sehr unmittelbare und oft tragende Rolle bei militärische Operationen" zugeschrieben. ${ }^{72}$ Insgesamt wurde 1396 Datensätze gesammelt, dabei kamen 200 Berater und 528 Informanten zum Einsatz. ${ }^{73}$ So entstan-

\footnotetext{
${ }^{68}$ Ebd.

${ }^{69}$ Ebd. 82.

${ }^{70} \mathrm{Ebd}$.

${ }^{71}$ William Langer an Ralph Barton Perry, 23. 11. 1943, HUA, HUD 3139 Records of the ADHG, Box 48.

${ }^{72}$ Ralph Barton Perry an Josef Laurenz Kunz, 4. 10. 1943, HUA, HUD 3139 Records of the ADHG, Box 48.

${ }^{73}$ Abschlussbericht des ADHG Biographical Record Committee, 25. 5. 1945, HUA, HUD 3139 Records of ADHG, Box 44.
} 
den 178 biografische Aufzeichnungen über Österreicher, wobei eine große Mehrheit Personen aus Wien behandelte. ${ }^{74}$

Ralph Barton Perry verfasste ein Memorandum über den Umgang mit den gesammelten biografischen Aufzeichnungen und wies darauf hin, dass diese Aufzeichnungen als streng geheim galten: „In Washington werden die Akten der Biographical Records Section, egal ob auf Karteikarten oder in Dokumenten, in schweren Tresoren mit Kombinationsschlössern aufbewahrt, in Räumen, die durchgehend von Mitarbeitern beaufsichtigt werden, solange die Tresore geöffnet sind. Diese Räume befinden sich wiederum in einem Gebäude, das unter 24-stündiger Bewachung steht und zu dem nur Mitglieder der Abteilung oder andere autorisierte Personen mit einem Sonderpass Zugang erhalten. [...] Der Zugang zu den Akten der Sektion ,Biografische Aufzeichnungen' ist nur Mitgliedern des Sektionspersonals oder ordnungsgemäß bevollmächtigten Personen für klar definierte Zwecke möglich. [...] Die Konsultation von Material für biografische Aufzeichnungen muss in den Büros der Sektion unter der Aufsicht eines Mitarbeiters erfolgen. Nur Angehörige des Sektionspersonals dürfen sich in dem Raum, in dem die Tresore aufbewahrt werden, aufhalten. Alle Mitarbeiter erfahren vor Dienstantritt eine besondere Sicherheitsuntersuchung. Die Kombinationen der Tresore sind nur drei verantwortlichen Mitarbeitern bekannt. ${ }^{\text {"75 }}$

Obwohl in diesem Memorandum zunächst der streng geheime Charakter der Information betont wird, offenbart es auch den Umstand, dass es der ADHG manchmal an der nötigen Diskretion in Sachen Spionage fehlte. Durch die Zusendung

\footnotetext{
${ }^{74}$ Liste der Consultants and Sources from Austria, HUA, HUD 3139 Records of the ADHG, Box 44.

${ }^{75}$ Ralph Barton Perry, Information Letter for Sources on Biographical Records, HUA, HUD 3139 Records of the ADHG, Box 44.

${ }^{76}$ B.M. Kellmurray an Ralph Barton Berry, 6. 10. 1943, HUA, HUD 3139 Records of the ADHG, Box 48.
}

dieses Schreibens an mögliche Quellen und Berater wurden mehr Informationen über diese geheime Operation zugänglich gemacht, als die kontaktierten Informanten benötigten. Diese schriftlichen Informationen hätten leicht abgefangen oder von einem Empfänger an einen gegnerischen Agenten weitergegeben werden können. Ralph B. Perry beabsichtigte höchstwahrscheinlich, die potenziellen Informanten dahin gehend $\mathrm{zu}$ beruhigen, dass alle Vorkehrungen getroffen wurden, um die Informationen und damit die in diesen biografischen Aufzeichnungen genannten Personen zu schützen. Diese Zusicherung war notwendig, da Berichte über persönliche Besuche bei Informanten besagten, dass diese sich nicht wohl zu fühlen schienen, Informationen über mögliche Kollaborateure an das OSS weiterzugeben. ${ }^{76}$ Emmy Crysler Rado von der New York Branch berichtete auch, dass die befragten Flüchtlinge ängstlich und nervös waren und sie vermuteten, dass Rados OSS-Büro eine von der Gestapo inspirierte Organisation wäre und Informationen nach Deutschland weitergeben würde. ${ }^{77}$

Die Handlungen durch die ADHG gefährdeten die möglichen Kollaborateure mehr als nötig, indem sie einen sensiblen Teil der Hintergrundstruktur des OSS in Washington skizzierten. Darüber hinaus wurde die Kennzeichnung der verwendeten vorgedruckten Formen durch das ADHG vom OSS als problematisch angesehen, da die zuständige OSS-Sektion und der volle Name der ADHG auf den gedruckten Formularen genannt wurden. ${ }^{78}$ Der ADHG wurde geraten, diese Beschriftung zu schwärzen oder den betroffenen Teil der Vordrucke abzuschneiden. ${ }^{79}$ Der kommissarische Leiter der Sektion Biografische Aufzeich-

\footnotetext{
${ }^{77}$ MCINTOSH, Sisterhood of Spies 79.

${ }^{78}$ G.W. Cottrell an Ralph Barton Perry, 1. 10. 1943, HUA, HUD 3139 Records of the ADHG, Box 44. ${ }^{79}$ Ebd.
} 
nungen G. W. Cottrell, musste die ADHG anweisen, diese Vordrucke vollständig auszuscheiden. Zwar hatte ADHG die unerwünschten Informationen auf den Blättern geschwärzt, aber diese waren nach einer kurzen Untersuchung immer noch lesbar. ${ }^{80}$ Das OSS erkannte zu Recht die Möglichkeit einer Enttarnung durch die Kennzeichnung der Vordrucke, die auch noch vereinzelt in den CIA-Crest-Akten zu finden sind. Diese führten schließlich auch den Autor dieser Arbeit zu den ADHG-Akten. ${ }^{81}$

Zusätzlich führte die ADHG auch ihre eigenen ausgedehnten und detaillierten Aufzeichnungen über die Materialien, die nach Washington geschickt wurden. Diese Listen und Dateikarten wurden offensichtlich nicht nach den gleichen hohen Standards bewacht und begründeten eine weitere unnötige Verwundbarkeit für das Projekt und die beteiligten Personen. Die unnötige Korrespondenz mit möglichen Quellen führte sogar zu einer zusätzlichen Erörterung des genauen Umfangs der Zusammenarbeit mit dem OSS. Die zu untersuchenden Länder wurden genau eingeschränkt und die ADHG aufgefordert, die Korrespondenz auf ein Minimum zu beschränken. ${ }^{82} \mathrm{Wil-}$ liam L. Langer, der Direktor der Research und Analysis Branch des OSS, erinnerte Ralph B. Perry in einem Brief an die Notwendigkeit großer Sorgfalt beim Schutz von Informationen, die, wenn sie bekannt wären, Leben in feindlichen Ländern gefährden könnten. ${ }^{83}$ Eine Enttarnung der als kooperationsbereit bezeichneten Personen durch die Abwehr oder die Gestapo hätte dementsprechende Konsequenzen nach sich gezogen.

\footnotetext{
${ }^{80}$ G.W. Cottrell an Ralph Barton Perry 26.1. 1944, HUA, HUD 3139 Records of the ADHG, Box 44.

${ }^{81}$ Einige der ursprünglichen Vordrucke der ADHG können noch in den CIA-Crest-Dateien gefunden werden, andere wurden von OSS-Mitarbeitern umgeschrieben und daher können einige BRs im Original und in der umgeschriebenen Form gefunden werden. ${ }^{82}$ UNBEKANNT, HUA, HUD 3139 Records of the ADHG, Box 44.
}

\subsection{Sammeln der Informationen}

Die möglichen Informanten, die in den Vereinigten Staaten lebten, wurden meist per Post kontaktiert. Die ADHG entwarf und produzierte vier Vordrucke: FORM HA, FORM HB, FORM HC, FORM HE. Diese Formulare fungierten als Fragebögen und Anleitungen zum Ausfüllen der Vordrucke durch die potenziellen Informanten. ${ }^{84} \mathrm{Ab}$ gesehen von allgemeinen biografischen und politischen Informationen, die in FORM HA abgefragt wurden, suchte man nach Personen mit den folgenden Attributen, wie in FORM HC angegeben: „persönliche Integrität, Selbstlosigkeit und intellektuelle Ehrlichkeit; die einen guten Ruf genießen und Einfluss in ihren Gemeinschaften ausüben; und die über Fachkompetenz in ihren Fächern oder Berufen verfügen" ${ }^{85}$

Die dritte und vierte Vorlage (FORM HB, FORM HE) wurden übermittelt, um andere mögliche Quellen in Erfahrung zu bringen. Die kontaktierten Quellen, die andere mögliche Informanten nannten, wurden dann zusätzlich als Berater geführt. Eine retournierte und ausgefüllte FORM HA, die nun den eigentlichen biografischen Datensatz enthielt, wurde an das OSS in Washington weitergeleitet. Die sonstige Korrespondenz mit dem Informanten blieb dagegen bei der ADHG. Nur durch die Kombination der CIACrest-Akten und des Materials in den Archiven der Harvard University ist es daher möglich, einen umfassenden Überblick über das Material zu gewinnen, insbesondere wenn es um die Identifikation einzelner Informanten geht.

\footnotetext{
${ }^{83}$ William L. Langer an Ralph Barton Perry, 1. 5. 1943, HUA, HUD 3139 Records of the ADHG, Box 48.

${ }^{84}$ Form HA, HUA, HUD 3139 Records of the ADHG, Box 44.

${ }^{85}$ Form HC, HUA, HUD 3139 Records of the ADHG, Box 44 .
} 
Es gab auch neue Informanten, die außerhalb von Boston lebten und bei denen die Annahme bestand, dass sie über Informationen von großem Interesse verfügten. Diese wurden dem OSS für einen persönlichen Besuch und ein Interview vorgeschlagen. ${ }^{86}$

Die Interviewer, die die Anfragen für das Biographical Records Project durchführten, schickten auch Berichte über ihre Einsätze zurück. In diesen mission briefings wurde erwähnt, dass die Informanten dazu neigten, die Verantwortung an andere Personen weiterzugeben, indem sie den Interviewern andere Personen als mögliche Informanten nannten. ${ }^{87}$ Ein Interviewer beschrieb die allgemeine Situation wie folgt: „Wenn eine Person als Informant besucht wird, stellt er sich in der Regel als viel williger heraus, als Berater zu helfen [...]. Dies ist auf Druck bei der Arbeit, auf eine ausgedrückte, aber oft nicht vorhandene patriotische Haltung zurückzuführen und manchmal auf den Verdacht, dass die Nennung seines Namens früher oder später Probleme ans Licht bringen könnte, für die er verantwortlich sein könnte. “88

Es überrascht nicht, dass sich nicht alle Beteiligten im Zusammenhang mit Geheimdienstaktivitäten wohl fühlten; daher führte die New York Branch einen dreistufigen Ablauf des Interviews ein. ${ }^{89}$ Die Informanten wurden langsam vorbereitet, indem sie mehrere Interviews absolvierten. Das erste Gespräch zielte darauf ab, das Vertrauen aufzubauen; Informationen sollten erst ab dem zweiten Treffen erhoben werden. 90

Die Kosten der ADHG für das Projekt wurden vom OSS übernommen und betrafen Büroperso-

\footnotetext{
${ }^{86}$ T. G. Brown an Mr. G. W. Cottrell, HUA, HUD 3139 Records of the ADHG, Box 44.

${ }^{87}$ B. M. Kellmurry an Ralph Barton Perry, 6. 10. 1943, HUA, HUD 3139 Records of the ADHG, Box 48.

${ }^{88}$ Ebd.

${ }^{89}$ MCINTOSH, Sisterhood of Spies 80.

${ }^{90}$ Ebd.

${ }^{91}$ Biographical Records Projektausgabenkonto für Juli 1944, HUA, HUD 3139 Records of the ADHG, Box 44.
}

nal und Aufwendungen wie Telefon- und Portokosten. Die Quellen und Berater erhielten keine Abgeltung für ihre Arbeit.91

\subsection{Einige Quellen und Berater}

\subsubsection{Hans Kelsen}

Kelsen wurde 1881 in Prag geboren und zog 1883 mit seiner Familie nach Wien. Er studierte Rechtswissenschaften an der Wiener Fakultät und begründete die Reine Rechtslehre. Kelsen wird ein großer Einfluss auf den Aufbau und teilweise auch auf Inhalte der österreichischen Verfassung von 1920 zugeschrieben. ${ }^{92}$ Als Informant entwarf Hans Kelsen einen BR über Adolf Merkl, der als sein treuester Schüler und Anhänger seiner Theorien gilt. ${ }^{93}$ Es ist unklar, wer Kelsen als mögliche Quelle erstmals empfahl. Er war jedenfalls kein Unbekannter an der Harvard University, von der er eine Einladung zur 300. Jahrestagung der Gründung Harvards und zur Harvard Tercentenary Conference of Arts and Sciences erhielt. Ebenfalls erhielt er die Ehrendoktorwürde der Harvard University. ${ }^{4}$ Die Harvard Law School war auch die erste akademische Institution für den vertriebenen europäischen Gelehrten in den USA, wo er zum Teil finanziell von der Rockefeller Foundation unterstützt wurde und Oliver Wendel Holmes-Dozent für das akademische Jahr 1940/1941 war. ${ }^{95}$

Kelsen holte auch Ralph Barton Perrys wissenschaftliche Meinung zu seinem Aufsatz Value Judgments in the Science of Law ein. Warum genau Kelsen diese Expertise einholte, ist nicht ganz klar, aber Barton war in den USA als Philo-

\footnotetext{
${ }^{92}$ OLeCHOWSKI, Kelsens Beitrag zur Bundesverfassung 228.

${ }^{93}$ Staudigl-Ciechowicz, Von Adamovich bis Pfeifer 214.

${ }^{94}$ EHS, GASSNER, Hans Kelsen.

${ }^{95}$ Ebd.
} 
soph durchaus etabliert. Wie Thomas Olechowski in seiner Arbeit betont, hatte Kelsen Mühe, sich finanziell, sprachlich und wissenschaftlich an sein neues Umfeld anzupassen. Er zielte daher wahrscheinlich darauf $a b$, seine Arbeit für die anglo-amerikanische Sphäre bestmöglich zu adaptieren..$^{96}$

Taylor Starck, Vorsitzender des Biographical Records Committee, empfahl Kelsen in einem Brief vom Juli 1943 als möglichen Informanten. ${ }^{97}$ Kelsen wurde im August 1943 erstmals von der ADHG wegen biografischer Daten kontaktiert.98 Nachdem diese keine Antwort erhalten hatte, wurde er im Oktober erneut kontaktiert und um eine Antwort gebeten, auch wenn diese negativ ausfallen sollte. ${ }^{99}$

Kelsen antwortete mi einem kurzen Brief:

„Dear Prof Taylor,

with reference to your letter of October 5th, I am sending you enclosed a biographical record concerning Professor Adolf Merkl of the University of Vienna. He is the only person I know who fulfils the requirements specified in form $\mathrm{HC}$.

I knew, of course, many persons who, at the time before the Nazis came into power, were antiNazi. But only of Merkl I am sure that he has not changed his mind. He was one of my best students, and later on one of my colleagues at the Law Faculty in Vienna.

Very sincerely yours, Hans Kelsen"

Wie bereits ausgeführt, befindet sich der von Hans Kelsen verfasste BR zu Adolf Merkl in den Unterlagen des CIA-Crest-Progrmms. Er enthält folgende Informationen

„Merkl, Dr. Adolf: Former professor of law, University of Vienna; probable address: Grinzinger Allee 43A, Vienna / born in Vienna, 23 march

\footnotetext{
${ }^{96}$ OlechOWSKI, Kelsen in Berkeley 60.

${ }^{97}$ Taylor Starck an Ralph Barton Perry, 14. 7. 1943, HUA, HUD 3139 Records of ADHG, Box 44.

${ }_{98}$ Taylor Starck an Hans Kelsen, 5. 10. 1943, Kasten 48.

${ }^{99}$ Taylor Starck an Hans Kelsen, 25. 10 .1943, Kasten 48.

${ }^{100}$ Hans Kelsen, OSS Biographical Record H-1661 on Adolf Merkl 4,
}

[sic!] 1890; Aryan; married; Catholic / professor of administrative law at the University of Vienna from 1928 until dismissal by Nazis in 1938 / one of the most outstanding representatives of field of jurisprudence and administrative law; enjoys excellent standing in field; very high intellectual integrity; is a scholar rather than an administrator.

Source (49), Berkley.

American Defense, Harvard Group (CVI) Cambridge, 19 October 1943."100

Nachdem er seine Antwort und den BR über Merkl geschickt hatte, kontaktierte die ADHG Kelsen ein letztes Mal und drängte auf weitere Namen von Kooperationswilligen. Kelsen scheint nicht geantwortet und nur diesen einen BR über Merkl verfasst zu haben, was darauf hindeutet, dass er entweder das Vertrauen in die politische Integrität seiner ehemaligen Kollegen und Freunde verloren hatte oder er sie aufgrund mangelnder (administrativer) Fähigkeiten für die alliierten Bemühungen als nicht nützlich erachtete. In Merkls BR erwähnt Kelsen, dass Merkl eine "sehr hohe intellektuelle Integrität" habe, aber "Gelehrter und kein Verwalter" und daher laut Kelsen nicht wirklich geeignet sei. Sein ehemaliger Student und Freund Alfred Verdross von der Wiener Rechtswissenschaftlichen Fakultät, der im Laufe seiner Karriere mehrere bemerkenswerte Verwaltungspositionen innehatte und somit höchstwahrscheinlich hinsichtlich seiner Fähigkeiten geeignet gewesen wäre, wird von Kelsen nicht einbezogen. ${ }^{101}$ Verdross wurde 1942 in eine leitende Position an der Juristischen Fakultät Wien berufen und war Generalsekretär der Association Internationale Vitoria at Suarez. Wei-

www.cia.gov/library/readingroom/docs/CIA-RDP8200038R1000190017-4.pdf

(19. 10. 1943/14. 8. 2018).

${ }^{101}$ BusCH, Alfred Verdross 153. 
ters gründete er eine österreichische Niederlassung der internationalen Organisation The New Commonwealth. ${ }^{102}$ Kelsen erwähnte Verdross höchstwahrscheinlich nicht aufgrund des Unmutes über die erzwungene Übernahme der Zeitschrift für öffentliches Recht durch Verdross im Jahr 1934.

Ein weiterer Grund für die verzögerte Antwort könnte auch die Furcht vor der Gestapo gewesen sein. ${ }^{103}$ Merkl war bereits zwei Wochen nach dem Anschluss Österreichs im Jahr 1938 von der Gestapo aufgesucht worden, wobei ein verdächtiger Brief und sein Reisepass beschlagnahmt wurden. ${ }^{104}$ Dies ist ein deutlicher Hinweis darauf, dass die neuen Machthaber keinen positiven Eindruck von Merkl hatten. Auch wenn Kelsen von diesem Vorfall nichts gewusst hat, befürchtete er wohl zu Recht die möglichen Folgen für Merkl. Daher wollte er möglicherweise seinen treuen Freund durch seine Nennung nicht gefährden. Dass ihre Freundschaft nicht beeinträchtigt wurde, zeigt sich auch in einem Brief, den Kelsen nach dem Krieg im Namen Merkls schrieb. Er unterstützte Merkls Bemühungen bezüglich einer Rückkehr an die Universität Wien. ${ }^{105}$

\subsubsection{Josef Laurenz Kunz}

Josef Laurenz Kunz war ebenfalls ein ehemaliger Student und Kollege von Kelsen und wanderte 1932 in die Vereinigten Staaten aus, nachdem er an der Universität Wien unter anderem auch wegen seiner engen akademischen Beziehung zu Kelsen diskriminiert worden war. ${ }^{106}$ Im Jahr 1920 wurde Josef Kunz Rechtsberater Österreichs

\footnotetext{
${ }^{102}$ Ebd. 153.

${ }^{103}$ Ebd. 151.

${ }^{104}$ Staudigl-Ciechowicz, Von Adamovich bis Pfeifer 215.

${ }^{105}$ Ebd. 216.

${ }^{106}$ KAMMERHOFER, Josef Laurenz Kunz 244f.

${ }^{107}$ Ebd. 1f.

${ }^{108}$ KunZ, Völkerrecht und Gaskrieg 7.

${ }^{109}$ FosDICK, Geschichte der Rockefeller Foundation, Anlage II.

${ }^{110}$ Ebd. 3.
}

beim Völkerbund und bekleidete diese Position bis $1932 .{ }^{107}$ Kunz schrieb eine Monographie über chemische Kriegsführung während des Ersten Weltkriegs, welche er persönlich als Frontoffizier im Ersten Weltkrieg erlebt hatte. ${ }^{108}$ Während dieser Zeit muss er die Aufmerksamkeit von Allen W. Dulles auf sich gezogen haben. In der Zwischenkriegszeit war Dulles auch Rechtsberater der Delegationen für Waffenbeschränkung beim Völkerbund und sein Bruder John Foster Dulles war Präsident und Vorsitzender des Kuratoriums der Rockefeller Foundation. ${ }^{109}$ Kunz wurde 1932 erstmals als Rockefeller Fellow in die USA eingeladen, wo er 1934 zum ordentlichen Professor für Rechtswissenschaften an der University of Toledo (Ohio) ernannt wurde. ${ }^{110}$ Nach dem Ausbruch des Zweiten Weltkriegs wurde Allen Dulles OSS-Direktor für Europa. ${ }^{111}$

Als Kunz von der ADHG kontaktiert wurde, arbeitete er bereitwillig mit und verfasste einen BR über seinen engen Freund Alfred Verdross. Er fungierte auch als Berater, indem er andere mögliche Quellen in den USA benannte. ${ }^{112}$ Der folgende Brief von Kunz an die ADHG findet sich in den ADHG-Akten: ${ }^{113}$

"Dear Mr. Perry:

In Reply to your letter of October 4, I would like to state that as a man, who has devoted his life to the study of international law, I practically know all international lawyers of the world personally. But as I am a born Viennese and was from 1927 to 1932 lecturer in international law at Vienna University Law School, I will restrict myself to

\footnotetext{
${ }^{111}$ DULLES, Germany's Underground.

${ }^{112}$ Josef Laurenz Kunz, OSS Biographical Record FORM HA on Alfred Verdross, pag. 10-13 www.cia.gov/library/readingroom/docs/CIA-RDP8200038R001000200020-8.pdf

(15. 10. 1943/14. 8. 2018).

${ }^{113}$ Josef Laurenz Kunz an Ralph Barton Perry, 12. 10. 1943, HUA, HUD 3139 Records of the ADHG, Box 48.
} 
Austria and, according to the purpose of the Harvard Group, to scholars in my field. I am an American citizen, have been continuously in this country since 1932 and have since that time never been back to Europe. It is interesting to note, that of the professors of Vienna University Law School, apart from many who have died since 1932, a great number are no longer in Austria, but in the United States. Thus my great teacher and friend, Professor Hans Kelsen, who drafted the democratic Constitution of the Austrian Republic, is now Visiting Professor at the University of California, Berkeley. My Vienna University colleague, Dr. A. Lenhoff, 114 is now Librarian at the Buffalo University Law School and so on. This is true also with regard to younger men, who were, prior to 1932, students; some of them have been my disciples, as e.g. Dr. Hans Aufricht, now Secretary of the New York Commission to study the organization of peace.

One could say, that a large proportion of the $\mathrm{Vi}$ enna University Law School is now in this country. On the other hand, I learned that after the annexation of Austria by Germany 1938 the National Socialist regime has appointed young Germans (not Austrians) as full professors at Vienna Law School, often men who were, by no means, prominent scientifically, but unconditional adherents of the NSDAP.

Of the Vienna Law School of my time (prior to 1932) one of the few who is still there, is Prof. Alfred von Verdross; I have the honor to transmit in the Annex information concerning him.

I would be glad, if this information would be of some value to you. I need hardly say that I am always at your disposal and I am, dear Mr Perry, Very sincerely yours, Josef L. Kunz."115

\footnotetext{
${ }^{114}$ Lenhoff entwarf auch einen BR über Adolf Merkl; dieser befindet sich in den CIA-Crest files vor Hans Kelsen Beitrag über Adolf Merkl, OSS Biographical Record H-1661 on Adolf Merkl,
}

Sehr interessant ist, dass der BR von Kunz zu Verdross zusätzliche Informationen über Verdross enthält, etwa dass dieser offenbar 1939 versucht hatte, aus Österreich in die USA auszuwandern:

,[...] 7. Biography (brief): Has long belonged to staff of Vienna Univ. Law School since the early twenties, after having served a few years in the diplomatic service of the Austrian Foreign Office under the Republic of Austria. In the late twenties he was made full professor. His principal field is International Law. Accepted by the end of 1939 to continue to serve at Vienna University under the present regime, BUT was an enthusiastic adherent of the League of Nations, a scientific protagonist of compulsory international adjudication and of an advanced international organisation [...]

11. Political party or leaning

12. Evidence of discrimination on the part of the present regime: With Austrian Christian Social (that is, Catholic) Party. Immediately after the German annexation, Prof. Verdross was suspended from Vienna University for $1 \frac{1 / 2}{2}$ years, probably on account of his strong Catholic convictions and the fact that his brother, an aid to Chancellor Schuschnigg, was immediately sent to a concentration camp and was still there when I had the last letter from Prof. Verdross in 1940.

13. Intimate friends and associates: V. Additional comments: A personal friend of mine; was much with him between 1920 \& 1932, and in correspondence until 1940. During the time of his suspension he published nothing; his correspondence was under strict German censorship, so that he corresponded with me in Italian without signature and via Yugoslavia. Cf. on the discrimination against him the late Prof. J.W. Gerner's article in 'American Journal of International Law',

www.cia.gov/library/readingroom/docs/CIA-RDP8200038R1000190017-4.pdf

(19. 10. 1943/14. 8. 2018).

${ }^{115}$ Ebd. 
XXXIII, I; 1939, pp. 116-117. During the same time he begged me to see whether he could get a professorship in the United States, which proved impossible, due to the continuing depression. When he, at the end of 1939, accepted the offer of the new regime to continue at Vienna University, it must be borne in mind that he had failed to get a professorship in this country and that he was under strong financial strain, as he had to care not only for his own family and his old mother, but also for the whole family of his brother, sent to and detained in a German concentration camp.

Is personally known, esteemed and respected as a man and a scholar, by many leading American international lawyers, as e.g. Judge Manley O. Hudson of Harvard. Prof. Hans Kelsen, Univ. of Calif., political Science."116

Als enger Freund von Kunz und einer der wichtigsten Schüler Kelsens ist Verdross der einzige bekannte Gelehrte für Internationales Recht aus Wien, der kontinuierlich an der Wiener Rechtsschule tätig war. ${ }^{117}$ Untersuchungen von Oliver Rathkolb zeigen die Berührungspunkte mit den herrschenden Nazis bis hin zur systematischen Sympathie für das Regime in jener Zeit. ${ }^{118} \mathrm{Kunz}$ verteidigt Verdross, indem er erklärt, dieser habe sich nur mit den Nazis arrangiert, um seine Familie zu erhalten. Er schien sich sicher zu sein, dass Verdross noch zuverlässig war und in einer kommenden Militärregierung nützlich sein konnte. Im Gegensatz dazu erschien Kelsen dessen politische Ausrichtung vor und während des Krieges zweifelhafter. Nach dem Krieg gelang es Kunz, seine beiden Freunde Kelsen und Verdross $\mathrm{zu}$ versöhnen. ${ }^{119}$ Bemerkenswert ist auch, dass Alfred Verdross tatsächlich Teil des politischen

\footnotetext{
${ }^{116}$ Josef Laurenz Kunz, OSS Biographical Record FORM HA on Alfred Verdross, pag. 10-13 www.cia.gov/library/readingroom/docs/CIA-RDP8200038R001000200020-8.pdf

(15. 10. 1943/14. 8. 2018).

${ }^{117}$ BusCH, Der Kreis um Kelsen 153.

${ }^{118}$ RATHKOLB, Rechts- und Staatswissenschaftliche Fakultät 216ff.

${ }^{119}$ KAMMERHOFER, Josef Laurenz Kunz $246 f$.
}

Arms der O5-Widerstandsgruppe wurde, dem Provisorischen Österreichischen Nationalkomitee (POEN). Die O5 und ihr politischer Arm standen wiederum in Kontakt mit Fritz Molden, einem der wichtigsten OSS-Agenten von Allen Dulles in Österreich. ${ }^{120}$ Russische Beamte vermuteten, dass die POEN sogar von den amerikanischen und britischen Geheimdiensten geschaffen worden waren. ${ }^{121}$

In einem zweiten Brief an die ADHG nennt Kunz weitere Akademiker, die damals in den USA lebten und als Informanten eingesetzt werden könnten. Diese Liste enthielt den Namen des möglichen Informanten, die Position vor dem Krieg und seine aktuelle Adresse in den Vereinigten Staaten. Diese Liste bestand aus den folgenden Namen: Erich Hoor, Leo Gross, Erich Hula, Hans Herz, Wolfgang H. Kraus, Erich Voegelin, Emil von Hofmannsthal und Maximilian Kößler. ${ }^{122}$

Nach handschriftlichen Ergänzungen eines unbekannten Autors auf dem Brief wurden mehrere der vorgeschlagenen Personen zu Quellen und/oder Beratern des BRP.

\subsubsection{Otto Benesch}

Benesch war akademisch nicht mit Kelsen und seinem Kreis verbunden, aber er war einer der aktivsten österreichischen Akademiker im ADHG. Otto Benesch war Kunsthistoriker und wurde 1947 Direktor des Albertina Museums. ${ }^{123}$ 1941 war er Dozent an der Harvard Summer School und später Angestellter am Fogg Art Museum. ${ }^{124}$ Innerhalb der ADHG war er Mitglied

\footnotetext{
${ }^{120}$ MUgraUer, Bande von Gaunern 101ff.

${ }^{121}$ STELZL-MARX, Carl Szokoll 95.

122 Josef Laurenz Kunz to Thomas G. Braun 5. 11. 1943, HUA, HUD 3139 Records of the ADHG, Box 13.

${ }^{123}$ FeICHTINGER, Zwischen den Kulturen 434.

${ }^{124}$ Ebd.
} 
des Führungsgremiums ${ }^{125}$ und wie bereits erwähnt, trugen Benesch und seine Frau auch zur Arbeit des Denkmalschutzausschusses bei. Innerhalb des BRP war er auch einer der produktivsten Berater, da er für 125 biografische Aufzeichnungen verantwortlich war. ${ }^{126}$ Seine Arbeit und seine Loyalität gegenüber amerikanischen Interessen schienen von US-Beamten sehr geschätzt worden zu sein; nach dem Krieg gehörten Otto Benesch und Ferdinand Czernin zu den ersten, die für eine rasche freiwillige Rückreise nach Österreich empfohlen wurden. (siehe unten in Kapitel 2.6.5). ${ }^{127}$

\subsubsection{Albert Ehrenzweig Junior}

Albert Rudolf Ehrenzweig war Richter und Universitätsdozent in Österreich. 1938 floh er mit seiner Familie aus Österreich, wo ihm im Juni 1939 der akademische Abschluss aberkannt wurde. ${ }^{128}$ Er emigrierte in die USA, kehrte aber im Gegensatz zu seinem Vater, einem Universitätsprofessor an der juristischen Fakultät in Wien und ebenfalls ehemaligem Richter, nach dem Krieg nie wieder zurück. ${ }^{129}$ Von 1942 bis 1944 war er Mitglied der Law Revision Commission of New York, arbeitete für eine renommierte Kanzlei in New York und lehrte von 1947 bis zu seinem Tod 1974 an der Berkeley Law School. ${ }^{130}$ Ein Schreiben von Albert Ehrenzweig findet sich in den ADHGAkten und enthält eine Studie über das österreichische Justizsystem im Zusammenhang mit Nachkriegsfragen. Der Brief von Ehrenzweig gibt einen Einblick in dessen Ansichten über die österreichische Justiz und ihre Vertreter. ${ }^{131}$ Weiters enthält der Brief einen kurzen historischen Überblick und beschreibt die politischen und persönlichen Hintergründe österreichischer Richter:

„Dear Frederic:

\footnotetext{
${ }^{125}$ Liste der Mitglieder des Lenkungsausschusses, an welche wöchentliche Berichte gesendet werden HUA, HUD 3139 Records of the ADHG, Box 86.

${ }^{126}$ Liste der Consultants, HUA, HUD 3139 Records of the ADHG, Box 44.

${ }^{127}$ EPPEL, Österreicher im Exil 2, 710.
}

Realizing that from now on, more than ever before, you will be concerned with problems of European reconstruction, I should like to tell you about a thought which occurred to me recently in this connection.

You know that before the invasion I served in the courts of Upper and Lower Austria for ten years, five years of which I worked as a judge in rural as well as metropolitan communities. Since my period of service included several years of political unrest, the close contact I had with all parts of the population has acquainted me to a considerable extent with both the typical setup of the political life in those communities, and with the moral and political attitude of many individuals, and administrative employees. I should of course be only too glad to put these experiences to the use of any governmental agency that might be interested in collecting data.

There is another matter that has been worrying me a great deal. There seems to be a danger that in any plan of reconstruction Austria will be treated as just another region of Germany - I do not mean from the standpoint of merit or demerit, but from a merely technical point of view. Such a procedure would involve a waste which the United Nations can ill afford in a situation which will require the utilization of all available constructive forces. To cut my story short: Austria can claim an administrative asset having no equal in any other part of Central Europe, i.e. her judicial personnel, which in my opinion, would offer an ideal center around which to construct a system of any military or administrative government that might be deemed desirable.

While the judicial system in Germany has undergone several violent changes (swing to the left after the first war, and early reaction to the right),

\footnotetext{
${ }^{128}$ MAIR, Zivilverfahrensrecht 302f.

${ }^{129} \mathrm{Ebd}$.

${ }^{130}$ RIESENFELD, BARTON, HILL, Universität Berkeley.

${ }^{131}$ Albert Ehrenzweig an Frederic Hellin, 2. 1. 1944, HUA, HUD 3139 Records of the ADHG, Box 44.
} 
while moreover in the successor states of AustroHungary the German-speaking judges of the monarchy were largely replaced by native judges, appointed according to the prevailing political trend, Austria, until very recently (according to my knowledge until 1942), kept in office a homogenous stock of judges belonging to a body which in continuous regeneration can be traced back to the time of the monarchy.

The decisive feature of this development is the fact that this body survived the revolution of 1918, the leftist era of the socialist coalition, the Dollfuss, the Heimwehr and the Schuschnigg period, and to an astonishing extent the Hitler regime, - probably because the large majority of this body belonged to a political group which, practically extinct and totally powerless elsewhere, represented a curious mixture of liberal, anti-catholic, anti-socialist, anti-radical tendencies, an utterly colorless and harmless type of Biertisch-politics. But, aside of these not too commendable qualities, these men had, I should think, still have, one merit which has made them acceptable to all groups successively in power and has won them a tremendous confidence in all classes of the population: their absolute impartiality and incorruptibility, which in view of the intolerable financial and political conditions under which they developed (a young judge used to have monthly salary of $\$ 60$ ), can be explained only by the tremendous force of the uninterrupted tradition of a cast [sic!]. The fact that almost never a man was appointed judge, who had not at least an uncle in court service, certainly did not make for a particularly high-grade selection, but it made it unthinkable for any member of the cast [sic!] to dishonour his family and profession.

\footnotetext{
${ }^{132} \mathrm{Ebd}$.

${ }^{133}$ Biographical Records by OSS New York Branch on Rudolf Michler, Max Strassner, Julius Adolf Seitz, CIACREST

[https://www.cia.gov/library/readingroom/docs/CIARDP82-00038R001000200009-1.pdf]

(21. 10. 1943/14. 8. 2018).
}

Far remote as these Austrian judges may be from an ideal body of administrators, they represent a group of men who would administer the laws of the United States as faithfully as the lays of monarchic, republican and totalitarian Austria, and, most important, would command a popular confidence, which no other group of officials could claim, a confidence comparable probably only to that offered to the clerus in Italy.

I have drafted for myself a blueprint of a kind of administrative system based on the judiciary under Allied control, and should be happy to write you more about this, in case you should think it to be worth while [sic!].

Cordially Yours,

Albert Ehrenzweig." "132

Die OSS-Akten enthalten auch eine Reihe von biografischen Aufzeichnungen über hochrangige österreichische Richter, die alle als echte Demokraten galten und für Nachkriegspläne sehr wertvoll waren. ${ }^{133}$ Die BRs wurden über folgendende österreichische Richter verfasst: Rudolf Michler, Max Strassner, Julius Stava und Adolf Seitz. ${ }^{134}$ Diese BRs wurden nicht über die ADHG gesammelt, da sie das Etikett der BRP-Niederlassung New York tragen. ${ }^{135}$ Die Quelle bleibt unbekannt, aber Ehrenzweig war laut seinem Brief in New York ansässig und es ist daher möglich, dass die New York Branch ihn dort persönlich interviewte. Ein Schreiben des OSS an die ADHG bestätigt, dass auch die New Yorker Niederlassung diesbezügliche Informationen sammelte. ${ }^{136}$ Eine Liste von schriftlichen Dokumenten über Gerichte, Schulen, Gewerkschaften etc. listet Ehrenzweig als Autor einer Studie über österreichische Gerichte auf. ${ }^{137}$ Die OSS-Akten enthalten ein internes Memorandum von Franz Neumann, in

\footnotetext{
${ }^{134}$ Ebd.

${ }^{135}$ Ebd.

${ }^{136}$ S. W. Cottrell an Taylor Starck, 20. 11. 1943, HUA, HUD 3139 Records of the ADHG, Box 44.

${ }^{137}$ Liste der Direktiven, HUA, HUD 3139 Records of the ADHG, Box 44.
} 
welchem eine Studie über das österreichische Justizsystem erwähnt wird, die als sehr wertvoll galt. ${ }^{138}$ Neumann, der Jurist, Politikwissenschaftler und führender Intellektueller war, arbeitete für das OSS im Bereich der Nachkriegsplanung. ${ }^{139}$ Das Handbook for Military Government of Austria enthält ebenfalls einen Anhang zum österreichischen Gerichtssystem. ${ }^{140}$

\subsubsection{Ferdinand Czernin}

Ferdinand Czernin war ein österreichischer Aristokrat und der Sohn von Ottokar Czernin, welcher der drittletzte Außenminister des österreichischen Kaisers Karl gewesen war. Die Außendienststelle der OSS betrachtete ihn als einen der 20 interessantesten politisch Protagonisten. Er war Vorsitzender der größten österreichischen politischen Exilgruppe - der Austrian Action mit rund 1500 Mitgliedern. ${ }^{141}$ Die Austrian Action wurde heimlich von Großbritannien unterstützt und finanziert. ${ }^{142}$ Als Vorsitzender der Austrian Action stand er bereits vor dem Beginn des BRP mit der ADHG in Kontakt. ${ }^{143}$ Czernin wurde von seinem Freund Otto Benesch empfohlen und sogar eingeladen, bei einer der Sitzungen des Lenkungsausschusses der ADHG teilzunehmen. Er reichte eine Liste von 17 möglichen Informanten in den USA ein, die bei der Bereitstellung weiterer biografischer Aufzeichnungen hilfreich sein konnten. ${ }^{144}$ Czernin führt in dem Schreiben, dem die Liste beigefügt ist, aus, dass die Austrian Action bereits versucht habe, eine eigene Liste zu organisieren, aber nicht sehr weit gekommen sei, da ihr die notwendige Unterstützung fehle:145

„Dear Mr. Perry,

\footnotetext{
${ }^{138}$ Franz L. Neumann an William B. Kip, 6. 2. 1945, confidential OSS Office Memorandum 147, https://www.cia.gov/library/readingroom/docs/CIARDP13X00001R000100370003-1.pdf,

(6. 2. 1945/14. 8. 2018).

${ }^{139}$ STIFTER, Zwischen Geistiger Erneuerung und Restauration 166.

${ }_{140}$ Österreich Military Government, Handbook, Anhang Nr. 32B.
}

excuse me please for answering your letter so belatedly. The fact of the matter is that we have tried to organize such a list as you are compiling on your own. However, lacking the necessary support we have not got very far. May I be permitted to make a suggestion, which I also made to Sara Wambaugh, namely that we nominate to you individuals of high standing who know the various professional groups they were connected with in Austria. I enclose a tentative list of people whom it may be worth your while to approach. Please let me know, whether this is any good to you and whether we can be of any further help. Yours very sincerely, for Austrian Action

Ferdinand Czernin."

Czernins Liste enthielt folgende Personen und deren Tätigkeitsgebiete:

“Municipality of Vienna: Dr. Hugo Breitner, 1175 Indian Hill Road Claremont, Cal., Dr. Siegfried Altmann, 600 W 218 Street, New York, N.Y.

Foreign Trade: Dr. Richard Schueller, 3204 Oxford Avenue, The Bronx, N.Y.

Ministry of Finance: Dr. Oscar Wollheim, Hotel Belmont Plaza, Lexington Ave. \& 49 Street, New York, N.Y.

Foreign Office: Dr. Martin Fuchs, 730 W, 183 Street, New York, N.Y.

Police: Dr. Richard Redler, 3480 University Street, Montreal, Canada.

Press: Ludwig Ullmann, 119 W 80 Street, New York, N.Y.

Timber and Forestry: Dr. Egon Glesinger, Albemarle Inn, Ashville, N.C.

Salt Mining: Felix Gerstmann, 143W 80 Street, New York, N.Y.

\footnotetext{
${ }^{141}$ EPPEL, Österreicher im Exil 247.

${ }^{142} \mathrm{Ebd}$.

143 TRAUSSNIG, Militärischer Widerstand 43.

${ }^{144}$ Ralph Barton Perry an Sara Wambaugh, 27. 11. 1943, HUA, HUD 3139 Records of the ADHG, Box 44.

${ }^{145}$ Ferdinand Czernin zu Ralph Barton Perry, 29. 11. 1943, HUA, HUD 3139 Records of the ADHG, Box 47.
} 
Schools: Maria Deutsch, 243 Riverside Drive, New York, N.Y.

Monuments \& Art: Prof. Dr. Otto Benesch, 283 Harvard Street, Cambridge, Mass.

Theatre: Ernst Lothar, Hotel Gladstone, 114 E 52 Street, New York, N.Y., Hans Jaray, Hotel Crydon, 12 E 86 Street, New York, N.Y.

Banks: Dr. H. Oppenheim, 36 W 89 Street, New York, N.Y.

Courts of Law: Dr. Robert Langer, 14 W 75 Street, New York, N.Y.

Lawyers: Dr. S. Geyerhahn, 9406, 34 Road, Jackson Heights, L.I.N.Y."

Diese Liste wurde nach Washington geschickt und in einem Brief ausdrücklich erwähnt, da die ADHG diese Liste für besonders wertvoll erachtete. ${ }^{146}$

Der Studie von S.W. Gould über Österreich ist der oben erwähnte Bericht von Ferdinand Czernin über Otto Habsburgs Geburtstagsfeier in New York beigefügt. Otto Habsburgs (politische) Aktivitäten wurden durch das OSS überwacht und auch Czernin scheint den Geheimdiensten über Habsburg berichtet zu haben (siehe auch 1.4.1)..$^{147}$

In einer von Otto Habsburg erstellten Liste über politisch aktive Österreicher, beschrieb dieser Czernin dem OSS gegenüber dagegen wie folgt: "Czernin ist keine sehr starke Persönlichkeit, er scheint in der Politik nicht sehr gut zu urteilen [...]."148

Ende September 1945 empfahl der Leiter der OSS-Außenstelle für eine Gruppe von 17 österreichischen Flüchtlingen eine rasche Prüfung einer möglichen Rückkehr nach Österreich ins Außenministerium, darunter Czernin und Benesch. ${ }^{149}$ Die Anforderungen an diese Personen umfassten

${ }^{146}$ T. G. Brown an G. W. Cottrell 25. 2. 1944, HUA, HUD 3139 Records of the ADHG, Box 44.

${ }^{147}$ Ferdinand Czernin, Bericht zum 30. Geburtstagsdinner von Otto Habsburg, 20. 11. 1942, HUA, HUD 3139 Records of the ADHG, Box 18.

${ }^{148}$ EPPEL, Österreicher im Exil 2243.
Folgendes: „[...] (c) have obtained reasonable familiarity with the American scene and American institutions, and (d) that they can be expected to remain sympathetic to the United States."

\subsubsection{Felix Kaufmann}

Felix Kaufmann wurde 1895 in Wien geboren, wo er als interdisziplinärer Wissenschaftler an der Universität Wien arbeitete und von Hans Kelsen wissenschaftlich stark beeinflusst wurde. ${ }^{150}$ Aufgrund seiner jüdischen Herkunft wurde er diskriminiert und musste aus Österreich fliehen, nachdem 1938 seine Lehrerlaubnis an der Universität Wien widerrufen worden war. Nach seiner Ankunft in den USA wurde er wissenschaftlicher Mitarbeiter an der New School for Social Research in New York und Mitherausgeber der Zeitschrift Phenomenology and Philosophical Research. ${ }^{151}$

Kaufmanns schriftliche Erklärung, nachdem er von der ADHG kontaktiert wurde, spiegelt erneut den Vertrauensverlust gegenüber seinen ehemaligen österreichischen Kollegen wider, die nicht aus dem Land geflohen waren, da Kaufmann nur eine einzige Person vorschlug, die er für geeignet hielt:

„Dear Professor Perry

After the receipt of your letter of October 4th I have earnestly considered whether I could suggest any of my former colleagues of the University of Vienna who is still there, as absolutely reliable. There was only one man whom I would have dared to recommend and so I tried to get news about him. Today I received the news of his death. I am very sorry that I cannot provide you with a positive answer.

With best regards,

I am,

\footnotetext{
${ }^{149}$ Ebd. 710.

${ }^{150}$ KRISTOFERISCH, Kreis um Hans Kelsen 153.

${ }^{151}$ Ebd. 156.
} 
Yours sincerely,

Felix Kaufmann". ${ }^{152}$

Der Vorsitzende des Ausschusses für Biografische Aufzeichnungen fragte Kaufmann noch nach dem Namen der Person, für den Fall, dass der Name bereits in einer Akte stünde, ohne dass der Tod des Betreffenden darin vermerkt wäre. ${ }^{153}$ In einem zweiten kurzen Brief nannte Kaufmann Richard Strigl als den verstorbenen Kollegen, den er hatte vorschlagen wollen. ${ }^{154}$

\subsubsection{Leo Gross}

Leo Gross wurde in Galizien als Sohn eines jüdischen Kaufmanns geboren. Seine Familie zog nach Wien, wo er an der juristischen Fakultät in Wien studierte und ebenfalls Schüler von Hans Kelsen war. ${ }^{155}$ Er war auch ein Stipendiat der Rockefeller Foundation, was ihm ein Studium an der London School of Economics, der Columbia University in New York sowie der Harvard University ermöglichte, wo er 1931 einen Abschluss als Doktor der Juridical Science erhielt. 1935 begann er für das International Institute of Intellectual Cooperation in Paris zu arbeiten -, dem Vorgänger der UNESCO im Völkerbund. Nach einer abenteuerlichen Flucht aus Europa im Jahr 1940, begann er eine erfolgreiche akademische Karriere in den USA. Zu seinen dortigen Stationen gehörten eine Professur für Internationales Recht an der Fletcher School of Law and Diplomacy, eine Beraterstelle für die UNRAA (1943-1944) und die Rechtsabteilung der UNO (1947-1948). Er wurde Professor am US Naval War College (1954-1955) und 1956 Vorstandsmitglied der American Society of International Law. Beim American Journal of International Law, in dem er auch die meisten seiner wissenschaftlichen Arbeiten in

\footnotetext{
${ }^{152}$ Felix Kaufmann an Ralph Bartauf Perry, 31. 12. 1943 HUA, HUD 3139 Records of the ADHG, Box 48.

${ }^{153}$ Brief von Taylor Starck an Felix Kaufmann, 6. 1. 1944, HUA, HUD 3139 Records of the ADHG, Box 48. ${ }^{154}$ Brief von Felix Kaufmann an Professor Starck 17. 1. 1944, HUA, HUD 3139 Records of the ADHG, Box 48. ${ }^{155}$ KAMMERHOFER, Kreis um Hans Kelsen 115.
}

englischer Sprache veröffentlichte, wurde er Mitglied des Verlagsvorstands. Das Foreign Service Institute des US-Außenministeriums ernannte ihn von 1957 bis 1968 zum Dozenten.

In Bezug auf das BRP wurde Leo Gross 1943 von einem Interviewer persönlich besucht und kurz befragt. ${ }^{156}$ Während seines Interviews stellt der Interviewer fest, dass Leo Gross „ein wenig unruhig schien, wenn er gebeten wurde, bei dieser Arbeit zu helfen [...] er hat mir den Eindruck vermittelt, dass er weder sich selbst noch seine Freunde in Europa an Kriegsaktivitäten beteiligen wollte." ${ }^{157}$ Offensichtlich litten die Interviewer, die an der Universität Harvard arbeiteten, unter den gleichen Problemen wie die New Yorker Abteilung, jedoch wurde die dort entwickelte dreistufige Interviewtechnik offenbar nicht angewendet.

\subsubsection{Gottfried Haberler}

Gottfried Haberler wurde 1900 in Purkersdorf bei Wien geboren und studierte Wirtschafts- und Rechtswissenschaften in Wien, London und Harvard. ${ }^{158}$ Nachdem er außerordentlicher Professor in Wien geworden war, beendete er dort seine akademische Karriere bereits in den 1930er Jahren, indem er Gastdozent an der Harvard University (1931/32) und Experte des Völkerbundes in Genf (1934-1936) wurde. Seine Frau galt als „Mischling zweiten Grades" nach den Nürnberger Rassengesetzen. ${ }^{159}$ Er emigrierte daher schon vor dem Anschluss, im Jahr 1936, in die USA, wo er 1943 eingebürgert wurde. Seine akademische Karriere in den USA war sehr erfolgreich, da er Professor für internationalen Handel an der Harvard University wurde und mehrere andere hochrangige akademische und politische Positionen innehatte. Er wurde Chief Consultant Officer

\footnotetext{
${ }^{156}$ B. M. Kellmurry an Ralph Barton Perry, 6. 10. 1943, HUA, HUD 3139 Records of the ADHG, Box 48.

${ }^{157}$ Ebd.

${ }^{158}$ HuBER, Gottfried Haberler.

${ }^{159}$ Ebd.
} 
of Price Administration (1941), Berater im Board of Governors des Federal Reserve System of the USA, Vizepräsident (1949) und Präsident (1962) der American Econometric Society, Mitglied und später Präsident (1950/51) der International Economic Society und des National Bureau of Economic Research in New York. Haberler erhielt auch internationale Ehrendoktorwürden zahlreicher Universitäten. ${ }^{160}$ Er war ein weltbekannter und sehr einflussreicher Experte für internationale Wirtschaft und Mitglied des berühmten Mises-Kreises. ${ }^{161}$

In Bezug auf das BRP empfahl Ralph Barton Perry dem OSS, Haberler an seiner Heimatadresse in Washington $\mathrm{zu}$ besuchen, und erwähnte, dass dieser bereits einen Datensatz eingeschickt und weitere Vordrucke zum Ausfüllen angefordert habe. ${ }^{162}$ Gottfried Haberler wurde 1944 von Sara Wambaugh auch als Interviewer für das BRP vorgeschlagen und versuchte, Mitglieder der Federal Reserve zu interviewen. ${ }^{163}$ Neben der Zusammenarbeit mit dem OSS beim BRP war er auch Mitglied des Ausschusses für Probleme der Friedensregelung innerhalb der ADHG. Angesichts seines bisherigen Fachbereichs, der internationalen Ökonomie - zusammen mit seiner Erfahrung aus dem Völkerbund -, war er bestmöglich qualifiziert.

\section{Schlussfolgerung}

Die Aufzeichnungen der ADHG zeigen nicht nur das Engagement einer Gruppe führender Wissenschaftler und ihre Anstrengungen bei der Verteidigung der westlichen Demokratie, dem Schutz ihrer Heimat und der Wiederherstellung von Demokratie und Freiheit in Europa. Sie bie-

\footnotetext{
${ }^{160}$ Ebd.

${ }^{161}$ SALERNO, Gottfried Haberler.

${ }^{162}$ T. G. Brown an G. W. Cottrell, 1. 11. 1943, HUA,

HUD 3139 Records of the ADHG, Box 44.
}

ten auch einen Einblick in die Entstehung des ersten modernen Geheimdienstes und seiner Verwissenschaftlichung.

Das Biographical Record Project als Teil des US Civil Affairs Program hatte offensichtlich eine große Bedeutung für den Wiederaufbau der europäischen Demokratie. Es ermöglichte den Alliierten, vertrauenswürdige Partner in der unmittelbaren Nachkriegszeit zu finden. Zusammen mit anderen Informationen und wissenschaftlicher Forschung wurden diese Erkenntnisse in mehreren Guides und Büchern verwendet, die an alliiertes Personal verteilt wurden und in militärischen Kursen Verwendung fanden. Kelsen, Kunz und andere Akademiker aus Österreich beteiligten sich an den geheimen Vorbereitungen der US-Regierung für das Wiederaufleben der Republik Österreich nach dem Zweiten Weltkrieg.

Durch die Briefe und Notizen dieser Informanten wird deutlich, dass oft ein großer Vertrauensverlust gegenüber ehemaligen Kollegen und Freunden eingetreten war. Hans Kelsens und Richard Strigls Briefe an die ADHG stellen diesen Bruch wohl auf die eindringlichste Weise dar. Kelsen muss mehrere Personen gekannt haben, die die geforderten Anforderungen hätten erfüllen können. Leider scheint er bis 1943 den Glauben an die moralische und politische Integrität seiner ehemaligen Weggefährten und Freunde in Österreich verloren $\mathrm{zu}$ haben. Es kann als Ziel der zukünftigen Forschung betrachtet werden, die Mitwirkung österreichischer Exilanten an den Vorbereitungen zur Entnazifizierung sowie am Wiederaufbau der Republik Österreich und ihre mögliche Einflussnahme auf die damit zusammenhängenden Entscheidungen der amerikanischen Behörden genauer zu untersuchen.

${ }^{163}$ Taylor Starck an G. W. Cottrell, 10. 1. 1944, HUA, HUD 3139 Records of the ADHG, Box 44. 


\section{Korrespondenz:}

Stefan RASTL

Kleingarten Wasserwiese

Gruppe 5, Parzelle 18

1020 Wien

stefan.rastl@stud.sbg.ac.at

ORCID-Nr. 0000-0003-2437-8318

\section{Abkürzungen:}

$\begin{array}{ll}\text { ADHG } & \text { American Defense, Harvard Group } \\ \text { BR } & \text { Biographical Record } \\ \text { BRP } & \text { Biographical Records Project } \\ \text { COI } & \text { Coordinator of Information } \\ \text { DÖW } & \begin{array}{l}\text { Dokumentationsarchiv des } \\ \text { Österreichischen Widerstandes }\end{array} \\ \text { HUA } & \text { Harvard University Archives } \\ \text { OSS } & \text { Office of Strategic Services } \\ \text { POEN } & \begin{array}{l}\text { Provisorisches Österreichisches } \\ \text { Nationalkomitee }\end{array} \\ \text { UNRAA } & \text { United Nations Relief and } \\ & \text { Rehabilitation Administration }\end{array}$

Siehe auch das allgemeine Abkürzungsverzeichnis: [http://www.rechtsgeschichte.at/media/abk.pdf]

\section{Archivmaterial:}

Harvard University Archives (Cambridge, Mass.), HUD 3139 Records of the American Defense Harvard Group, Boxes 1, 4, 5, 7, 8, 9, 11, 13, 14, 18, 31, $34,44,45,47,48,72,73,86$.

CIA-CREST, OSS Collection [https://www.cia.gov/library/readingroom/collection/oss-collection] (18. 1. 2017/14. 8. 2018).

\section{Literatur:}

Jürgen Busch, Alfred Verdross, Ein Mann des Widerspruchs?, in: Franz-Stefan MeIssel u.a. (Hgg.), Vertriebenes Recht - Vertreibendes Recht (Wien 2012) 129-170.

Allen Welsh Dulles, Germany's Underground. The Anti-Nazi Resistance (o.O. 1947).

Tamara EHS, Miriam GASSNER, Hans Kelsen (18811973). Legal scholar between Europe and the Amer- icas http://www.transatlanticperspectives.org/entry.php?rec $=132$

(8. 11. 2012/14. 8. 2018).

Peter EPPEL, Österreicher im Exil. USA 1938-1945, Bd 2 (Wien 1999).

Johannes FEICHTINGER, Wissenschaft zwischen den Kulturen. Österreichische Hochschullehrer in der Emigration 1933-1945 (Frankfurt 2001).

Andreas HubER, Gottfried Haberler, Prof. DDr. Dr. h.c. [http://geschichte.univie.ac.at/de/personen/gottfried-haberler-prof-ddr-dr-hc]

(15. 2. 2018/14. 8. 2018)

Jörg KAMMERHOFER, Josef Laurenz Kunz, in: Robert WALteR u.a. (Hgg.), Der Kreis um Kelsen: Die Anfangsjahre der Reinen Rechtslehre (Wien 2008) 243-259.

Hans KRISTOFERISCH, Andreas ORATOR, Felix Kaufmann, in: Franz-Stefan MeIssel u.a. (Hgg.), Vertriebenes Recht - Vertreibendes Recht (Wien 2012) 153-174.

Josef Laurenz KUNZ, Gaskrieg und Völkerrecht (Wien 1927).

Julia MAIR, Das Zivilverfahrensrecht in den Jahren 1938 bis 1945. Unter besonderer Berücksichtigung der Schicksale der Zivilverfahrensrechtler der Universität Wien, in: Franz-Stefan MEISSEL u.a. (Hgg.), Vertriebenes Recht - Vertreibendes Recht (Wien 2012) 301-350.

Elizabeth P. McINTOSH, Woman of the OSS. Sisterhood of Spies (Annapolis 1998).

Manfred MUGRAUER, Eine „Bande von Gaunern, Schwindlern und naiven Leuten“; in: DÖW (Hg.), Jahrbuch des Dokumentationsarchiv des österreichischen Widerstandes (Wien 2016) 101-139.

Österreich. Military Government (Hg.), Handbook for Military Government in Austria (Wien 1945).

Thomas OLechOWSKI, Der Beitrag Hans Kelsens zur österreichischen Bundesverfassung, in: Robert WALter u.a. (Hgg.), Hans Kelsen: Leben-WerkWirksamkeit (Wien 2009) 211-230.

Thomas OlechOWSKI, Kelsen in Berkeley, in: BRGÖ 6 (2016) 58-73.

Oliver RATHKOLB, Die Rechts- und Staatswissenschaftliche Fakultät der Universität Wien zwischen Antisemitismus, Deutschnationalismus und Nationalismus 1938, davor und danach, in: Gernot HeIß u.a. (Hgg.), Willfährige Wissenschaft (Wien 1989) 197232.

Stefan A. RIESENFELD u.a., LAW: Berkeley http://content.cdlib.org/view?docId=hb1199n68c\&doc.view= frames\&chunk.id=div00037\&toc.depth=1\&toc.id= (2011/15. 8. 2018). 
Joseph T. SALERNO, Gottfried Haberler (1900-1995) [https://mises.org/profile/gottfried-haberler] (5. 8. 2018).

Kamila Staudigl-Ciechowicz, Von Adamovich bis Pfeifer: Eine Auseinandersetzung mit der Staatsrechtslehre an der Universität Wien in Zeiten der politischen Umbrüche der ersten Hälfte des 20. Jahrhunderts. in: Franz Stefan MeIsSEL u.a. (Hgg.), Vertriebenes Recht - Vertreibendes Recht (Wien 2012).

Barbara STELZL-MARX, Carl Szokoll und die Operation "Radetzky". Militärischer Widerstand in Wien 1945 im Spiegel sowjetischer Dokumente, in: DÖW (Hg.), Jahrbuch des Dokumentationsarchiv des österreichischen Widerstandes (Wien 2009) 95-113.
Christian H. STIFTER, Zwischen Geistiger Erneuerung und Restauration. US-amerikanische Planung zur Entnazifizierung und demokratischen Neuorientierung österreichischer Wissenschaft 1941-1955 (Wien 2014).

Florian TRAUSSNIG, Militärischer Widerstand von außen. Österreicher in US-Armee und Kriegsgeheimdienst im Zweiten Weltkrieg (Wien-Köln-Weimar 2016).

UNBEKANNT, History of the CIA www.cia.gov/about-cia/history-of-the-cia (10. 4. 2007/14. 8. 2018). 